\title{
IV.
}

\section{Zur normalen und pathologischen Morphologie der inneren Secretion der Bauchspeicheldrüse ${ }^{1}$ ).}

(Die Bedeutung der Langerhans'schen Inseln.)

(Aus dem Patholog.-anatom. Laboratorium von Prof. K. N. Winogradow in St. Petersburg.)

Von

Dr. med. L. W. Ssobolew.

(Hierzu Taf. IV u. V.)

Bei der mikroskopischen Untersuchung der Bauchspeicheldrüse bei einer anderen Arbeit wurde meine Aufmerksamkeit auf die in dieser Drüse vorkommenden Langerhans'schen Zellhaufen oder Inseln gelenkt. Diese Zellgruppen, die beim Menschen manchmal eine Grösse von $1 \mathrm{~mm}$ im Durchmesser erreichen, sind nicht selten schon mit blossem Auge auf dem Schnitte in Form von weisslichen, halb durchsichtigen Punkten wahrnehmbar. Beim Hunde beträgt ihr Durchmesser $0,3-0,35 \mathrm{~mm}$, hier gelang es mir daher nicht, sie mit blossem Auge zu erkennen. Auf gefärbten Schnitten treten die Inseln in Form von hellen Stellen hervor, auf denen, besonders beim Menschen, die Zellen weniger dicht angeordnet sind. Die Inseln sind meist von runder oder avaler Form, doch finden sich auch solche von unregelmässig gelappter Form. Die Zellen der Inseln sind polygonal und etwas kleiner, als die Zellen der die Inseln umgebenden Schaltstücke des Canalsystems des Pankreas; ihr Protoplasma ist blass, feingekörnt, der Kern auch gewöhnlich etwas kleiner, als der Kern der Drüsenröhrchen-Zellen, hat aber ein dichteres ChromatinNetz und färbt sich in Folge dessen intensiver; vereinzelte Kerne können auch verhältnissmässig gross sein, ja selbst grösser, als

1) Diese Arbeit wurde beendet und erschien im Druck in russischer Sprache als Inaug.-Dissert. im Frühling 1901, ihre Uebertragung ins Deutsche wurde verschiedener Umstände halber auf den December 1901 verlegt. 
$\stackrel{\Xi}{\grave{~}}$
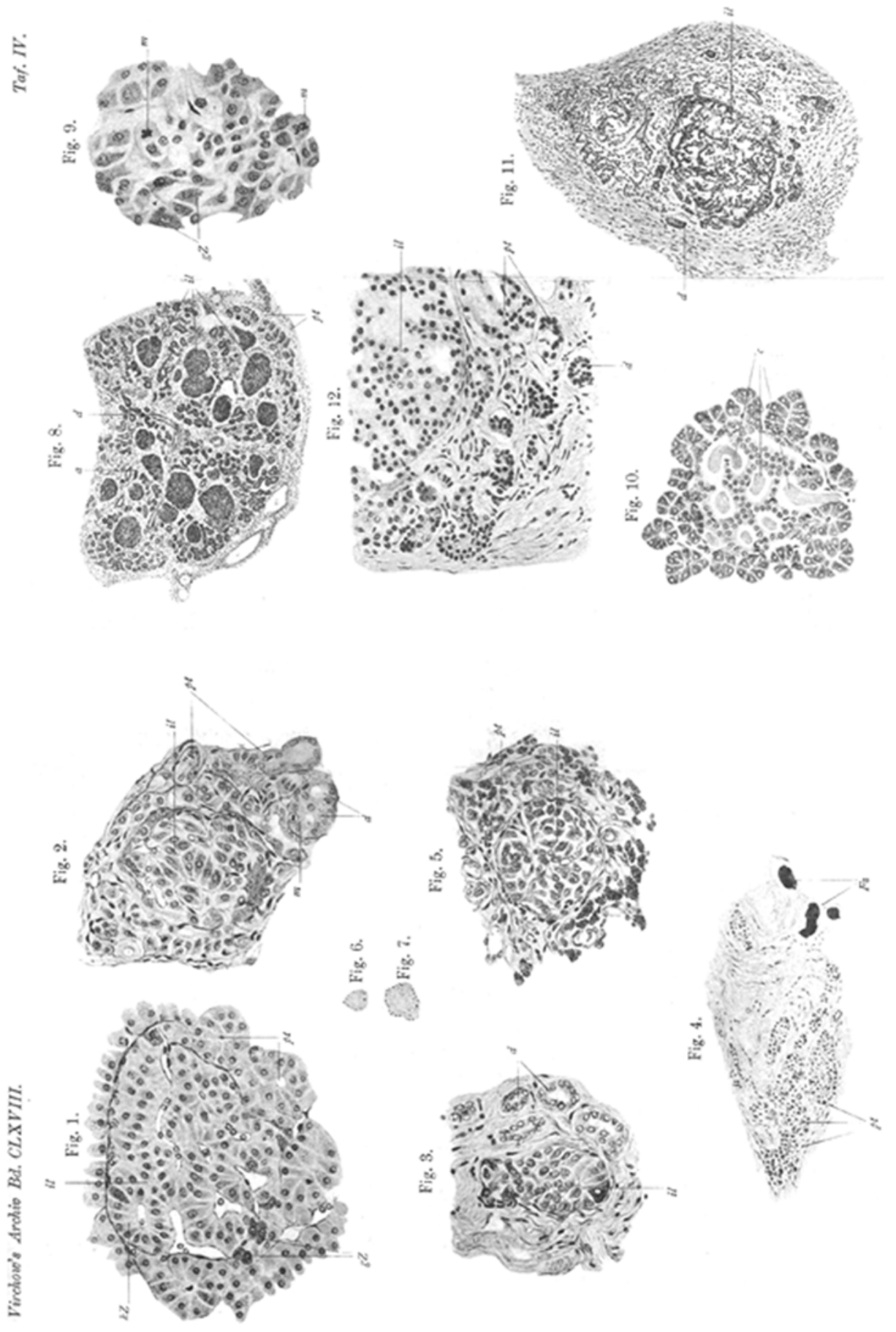
Fig. 13.

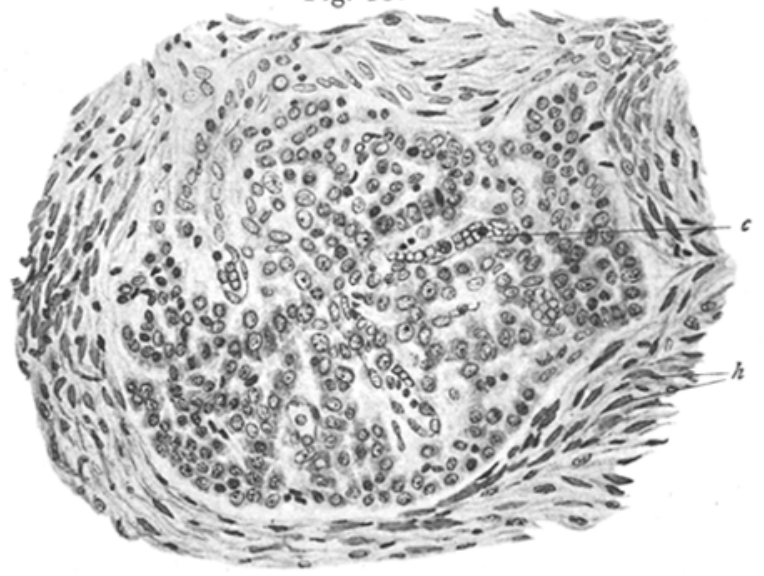

Fig. 14.

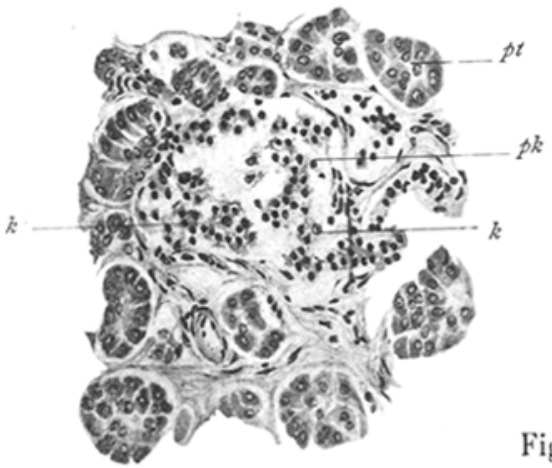

Fig. 15.

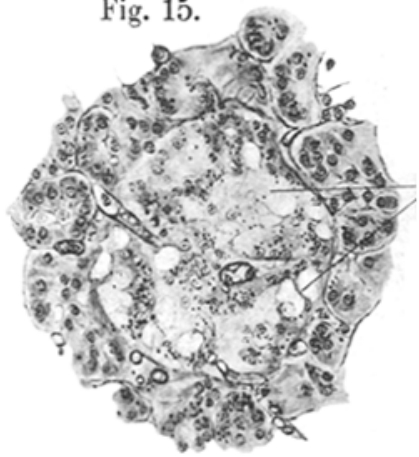

Fig. 16.

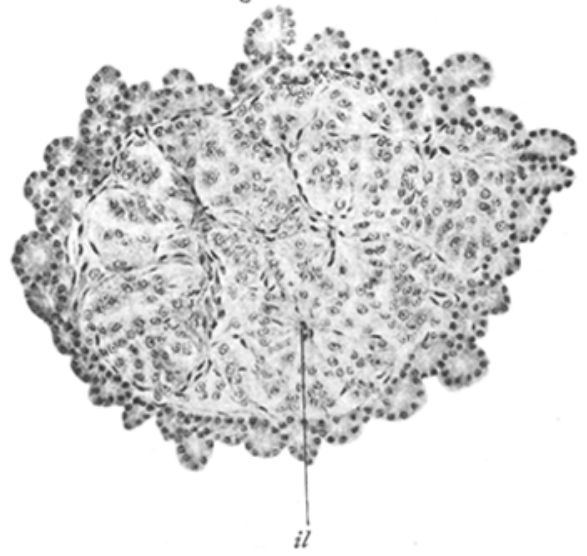

4d. net. J. Niknyot del. 
die Kerne der Drüsenröhrchen-Zellen. In den Kernen der Inselzellen findet man gewöhnlich 1-2 kleine Kernkörperchen. Bei der Mehrzahl der Säugethiere und bei den Vögeln haben die Inseln den gleichen Charakter, bei den Nagern aber (Kaninchen, Meerschweinchen) lassen sich deutlich 2 Arten von Zellen unterscheiden. Die eine Art der Zellen, die die centrale Hauptmasse der Inseln bildet, hat ein blasses, feingekörntes Protoplasma und einen ovalen Kern mit deutlich ausgesprochenem Chromatin-Netz; die Zellen der anderen Art, deren man auf einem Schnitt der Insel 5-10 trifft, sind grösser, von mehr runder Form, und in ihrem Protoplasma finden sich eine Menge Körnchen von grösserer Form, die durch Saffranin intensiv gefärbt werden; ausserdem bildet das Kern-Chromatin in den Zellen der zweiten Art, im Gegensatz zu den Zellen der ersten Art, wo es die Form eines deutlich ausgesprochenen Netzwerks hat, nur eine Kern-Membran und ein centrales grosses Chromosoma, das Netzwerk dagegen ist nur schwach angedeutet. Die zweite Art von Zellen findet sich vorzugsweise in den peripherischen Partien der Inseln.

Die Inseln bestehen aus geschlängelten Reihen in der Breite von $1-2$, selten mehr Zellen. Die Zellcolonnen sind durch Gefässcapillaren von einander getrennt, welche in den Inseln ein Capillarnetz bilden, das sich von einem Glomerulus nur durch das Fehlen einer Ausführungsarterie unterscheidet. Das Endothel der Capillaren liegt den Inselzellen unmittelbar an (Fig. 1 u. 10). Ausführungsgänge konnte ich in den Inseln nicht wahrnehmen. Manchmal täuscht das Lumen eines Capillargefässes einen Ausführungsgang vor, doch zeigte es sich bei der Untersuchung der benachbarten Schnitte, sowie der Schnitte von. blutreichen Drüsen, dass alle vorhandenen Lumina mit Blatkörperchen gefüllt waren. Ein Bindegewebs-Stroma haben die Inseln nicht, wohl aber trifft man bisweilen einige Bindegewebsfasern, die das in die Inseln eintretende Capillargefäss begleiten. Eine sie gegen das. übrige Gewebe abgrenzende Kapsel besitzen die Inseln auch nicht; bisweilen übernimmt die Rolle einer solchen Grenzmembran ein an der Peripherie der Insel sich hinziehendes Capillargefäss oder ein vereinzelter BindegewebsStrang. Meist grenzen die Inselzellen unmittelbar an die Membrana propria der Drüsenröhrchen, wobei sie manchmal Dank 
der Zartheit dieser Membran, deren Vorhandensein übrigens von einigen Autoren bestritten wird, und besonders während der Thätigkeit der Drüse, den Zellen der Drüsenröhrchen so ähnlich sein können, dass sie sich von diesen nur mit Mühe unterscheiden lassen. In schlecht fixirten Präparaten ist eine solche Unterscheidung überhaupt nicht möglich.

Die Grösse und die Zahl der Inseln ist für jede Thierart mehr oder weniger constant. Am besten entwiekelt sind die Inseln bei kleinen Thieren mit intensivem Stoffwechsel, so bei der Taube, dem Meerschweinchen; bei grossen Thieren, so z. B. beim Rinde, sind die Inseln verhältnissmässig klein und ihre Zahl gering. Die grössten Inseln finden wir beim Mensohen und beim Meerschweinchen.

Aus der eben angeführten Beschreibung der Inseln geht hervor, dass sie eine gewisse Aehnlichkeit haben mit Drüsen ohne Ausführungsgänge, wie es die Blutdrüsen, die Glandula suprarenalis; die Glandula parathyreoidea sind; diese letztere Drüse ähnelt auch in der Form der Zellen den Inseln und unterscheidet sich von diesen nur durch ihre bedeutendere Grösse. Andererseits ist wiederum bekannt, dass die Bauchspeicheldrüse auch die Function einer Blatdrüse hat, insofern, als einerseits - das Factum feststeht, dass eine Total-Exstirpation der Bauchspeicheldrüse bei Thieren unbedingt zu intensiven Erscheinungen des Diabetes führt, andererseits die Sections-Befunde von Diabetischen häufig Veränderungen der Bauchspeicheldrüse ergaben, die manchmal in völligem Schwund des Drüsenparenchyms bestanden, manchmal jedoch nur äusserst gering waren. Ausserdem ist noch bekannt, dass eine Unterbindung des Ausführungsganges der Bauchspeicheldrüse nicht zu Diabetes führt, obwohl es dabei zur Atrophie des Drüsenparenchyms kommt. Die beiden Facta lassen die Hypothese zu, dass gerade die Langerhans'schen Inseln das morphologische Substrat dieser besonderen, bei der Metamorphose der Kohlenhydrate eine Rolle spielenden Function der Bauchspeicheldrüse bilden. Diese Hypothese hebt auch den scheinbaren Widerspruch zwischen den beobachteten Erscheinungen auf. Zweck meiner Arbeit war es nun, diese Hypothese durch experimentelle und pathologisch-anatomische Untersuchungen auf ihre Richtigkeit hin zu prüfen. 
A. Experimenteller Theil.

I. Untersuchung der Bauchspeicheldrüse nach Unterbindung ihres Ausführungsganges.

Folgende Ueberlegung überzeugte mich von der Nothwendigkeit, Experimente mit Unterbindung des Ausführungsganges der Drüse anzustellen. Längst ist es ja bekannt, dass Drüsen oder Theile derselben, die durch einen Verschluss ihres Ausführungsganges der Möglichkeit beraubt sind, ihr Secret durch denselben zu entleeren, noch eine Zeit lang ihre secretorische Thätigkeit fortsetzen, wobei es zu einer Erweiterung der ausführenden Wege kommt, und dann erst atrophisch werden. Bei Unterbindung des Ausführungsganges der Bauchspeicheldrüse musste ich also als Folge-Erscheinung eine Atrophie ihres Verdaungs-Apparates erwarten, die Langerhans'schen Inseln dagegen, falls sie wirklich anatomisoh und functionell von dem Verdaungs-Apparat verschiedene Elemente darstellen, mussten erhalten bleiben. Auf diese Weise wird man genau das Verhältniss der Inseln zu den Ausführungsgängen feststellen können. Keinerlei künstliche Injectionen kommen im gegebenen Falle dieser Methode gleich. Nimmt man aber an, dass die Inseln Elemente darstellen, die in einiger Beziehung zu den Drüsenröhrchen stehen, so z. B. aus ermatteten abgearbeiteten Drüsenzellen bestehen ( $\mathrm{Lawdow}$ sky, Böhm und Dawydow), so müssen sie bei einer Atrophie der normalen Drüsenröhrchen gleichfalls zu Grunde gehen.

Auch folgende Ueberlegung überzeugte mich von der Nothwendigkeit einer mikroskopischen Untersuchung der Bauchspeicheldrüse nach Unterbindung ihres Ausführungsganges. Wie schon oben erwähnt wurde, steht das Factum fest, dass nur eine Total-Exstirpation der Drüse bei Thieren das Bild eines Diabetes hervorruft, die Unterbindung ihres Ausführungsganges dagegen wohl zu einer Atrophie der Drüse, doch ohne Zucker-Ausscheidung im Harn, führt. Diese beiden Facta lassen zwingender Weise den Gedanken anfkommen, dass nach Unterbindung des Ausführungsganges doch ein gewisses Etwas bleibt, dass die Entstehung des Diabetes verhindert. Es lässt sich allerdings noch annehmen, dass nach Unterbindung des Ausführungsganges die Function der Bauchspeicheldrüse nicht sofort sistirt wird, sondern 
allmählich erlischt, so dass andere Organe Zeit finden, sich dieser Function anzupassen und sie auf sich zu nehmen.

\section{Methodik.}

Die Thiere wurden durch Stich in die Medulla getödtet. Kleine Stücke der Drüse wurden für mikroskopische Untersuchung vorzugsweise in 3 Flüssigkeiten gebracht: 10 procentige FormalinLösung, Zenker'sche Flüssigkeit und nach Maximow modificirte, d. h. mit Sublimat versetzte Podwyssotsky'sche Flüssigkeit. In jede dieser Lösungen wurden für gewöhnlich 3 Stücke der Drüse, die dem Kopf, Schwanz und Körper entnommen wurden, gelegt. Zur Untersuchung der Zellkörnung benutzte ich die Fixation mit Formalin, Hermann'scher und Altmann'scher Lösung. Eingebettet wurden die Präparate vorzugsweise in Paraffin, theilweise aber auch, so die von Leichen entnommenen und die in Formalin gehärteten, in Celloidin. Die Schnitte von $3-7 \frac{1}{2} \mu$ Dicke wurden mit Wasser nach Gaule, mit Agar-agar oder Erweiss nach der japanischen Methode aufgeklebt. Zur Färbung benutzte ich Delafield'sches Hämatoxylin mit Eosin, Saffranin und Lichtgrün nach Benda, Heidenhain'sches EisenHämatoxylin; ferner färbte ich auch nach Altmann, Galeotti, Weigert (auf elastische Fasern).

Die Unterbindung des Ausführungsganges bei Kaninchen und Hunden wurde aseptisch unter Morphium- oder ChloroformNarkose ausgeführt. Beim Kaninchen mündet der Ausführungsgang der Bauchspeicheldrüse ungefähr $40-50 \mathrm{~cm}$ vom Pylorus entfernt und liegt frei im Mesenterium; gewöhnlich wurde ein Stück des Ausführungsganges zwischen zwei Ligaturen resecirt. Beim Hunde sind meist zwei Ausführungsgänge vorhanden, deren Isolation auf einer mehr oder weniger bedeutenden Strecke nicht gelingt; ich begnügte mich hier mit dem Durchschneiden des Ausführungsganges zwischen zwei Ligaturen. Die Durchgängigkeit des Ganges stellt sich dabei nach einiger Zeit wieder her und die Drüse regenerirt. Um diesen letzteren Umstand zu vermeiden, präparirte ich bei einem meiner Versuche die Drüsè vom Duodenum ab, wickelte sie in das Netz ein und versenkte sie so wieder in die Bauchhöhle. Bei der Katze mündet der Ductus Wirsungianus in den Darm gleich neben dem Gallengang, 
wodurch seine Isolirung stets erschwert wird, weswegen ich mich mit seiner Durchschneidung zwischen 2 Ligaturen begnügte; nach 20-30 Tagen wurde der Ductus Wirsungianus wieder durchgängig. An Meerschweinchen konnte ich wegen einer Epizootie, die unter diesen Thieren im hiesigen anatomischen Institut herrschte, keine Versuche anstellen. Operirt wurden von mir

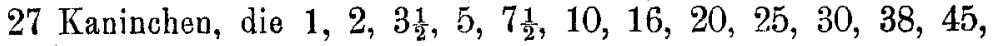
$60,68,75,90,105,120,150,200,400$ Tage nach der Operation getödtet wurden. Es trat dabei immer eine Erweiterung der grösseren Ausführungswege ein; die Drüse selbst verringerte sich vom 6. Tage an im Volumen, und zum Schluss blieben von ihr nur kleine weissliche Knötchen noch, die auch noch bis zum 150. Tage in allmählichem Schwinden begriffen waren; vom 150. Tage an wurde keine weitere Volumen-Abnahme beobachtet. Hunde wurden von mir 14 operirt und nach Ablauf von $5,10(2)$, $15(2), 20,25,30,35,40,50$ (2) und 60 (2) Tagen getödtet. Aus der Zahl dieser Versuche misslangen 5 insofern, als in einem Falle (10 Tage) beim Hunde der 3. accessorische Gang sich als nicht unterbunden erwies und die Drüse keinerlei Veränderungen zeigte. Ferner stellte sich in den Versuchen von 40, 50 (1) und 60 (2) tägiger Daụer die Durchgängigkeit des Ausführungsganges wieder her und die Drüse regenerirte. Bei den Hunden konnte man in der ersten Zeit eine starke ConsistenzVeränderung der Drüse constatiren, und erst vom 6.-11. Tage an begann der atrophische Process, der in den freien Enden der Drüse am stärksten ausgesprochen war. Die Erweiterung der Ausführungsgänge war gering. An Katzen wurden 12 Versuche angestellt, wobei die Thiere nach 4 (2), 15, 18 25, 60, 84, 90 und 100 Tagen getödtet wurden. Veränderungen der Drüse wurden jedoch nur in den ersten 5 Fällen gefunden, bei den übrigen Versuchen stellte sich die Durchgängigkeit des Ausführungsganges wieder her und die Drüse regenerirte. Bei den Katzen verlief der Process in gleicher Weise, wie bei den Hunden. Alle Thiere überstanden die Operation sehr gut. Bei den Kaninchen wurden keinerlei Verdauungs-Störungen beobachtet, was möglicher Weise sich dadurch erklären lässt, dạss diese Thiere eine grosse Anzahl von accessorischen Drüsen im Duodenum besitzen, die den Brunner'schen Drüsen ähnlich sind. Bei 
Hunden und Katzen wurden bei stark fetthaltiger Nahrung fettige Faeces beobachtet, auch magerten diese Thiere nach der Operation $a b$ und fielen im Gewicht, wogegen die Kaninchen an Gewicht zunahmen. Bei keinem der Thiere konnte Zucker im Harn nachgewiesen werden.

Ausserdem operirte ich 6 Kaninchen nach einem etwas modificirten Verfahren, - ich durchschnitt den Ausführungsgang, wobei jedoch nur das Darmende desselben unterbunden wurde, das andere Ende aber offen gelassen wurde. Ich nahm an, dass das normale Pankreas-Secret nicht verdauend auf das gesunde Peritonaeum wirken könne. Alle Kaninchen blieben am Leben

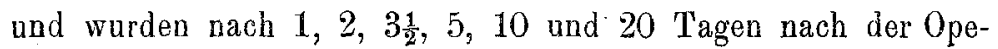
ration getödtet; das offene Ende des Ausführungsganges schloss sich bald (am 4.-5. Tage) durch Verklebung, das Bauchfell blieb unverändert, nur das die Ligatur des Darmendes umgebende Fettgewebe zeigte die Anzeichen einer Fettgewebs-Nekrose. Die Drüse atrophirte ebenso, wie nach der einfachen Unterbindung des Ausführungsganges.

Fassen wir nun die Resultate zusammen, die wir bei der mikroskopischen Untersuchung der Bauchspeicheldrüse nach Unterbindung ihres Ausführungsganges bei Kaninchen, Hunden und Katzen ${ }^{1}$ ) erhalten haben, so sehen wir, dass diese Resultate im Allgemeinen dieselben sind, und zwar unterliegt in der Drüse der Apparat einer Atrophie, der wit den Ausführungsgängen in innigem Zusammenhange steht, und augenscheinlich ausschliesslich der Verdauung dient, es sind dies die Drüsen-Röhrchen, erbalten dagegen bleibt ein anderer Apparat, die Langerhans'schen Inseln. Dabei wird allem Anschein nach anfangs nur die Secretion der Drüse stark herabgesetzt, die Zellen jedoch bleiben erhalten, bezw. sie nehmen beim Kaninchen den Charakter der Zellen des Hunger-Pancreas an, dann aber treten beim Kaninchen vom 3. Tage, beim Hunde vom 3. Tage, bei der Katze vom 5. Tage an die Anzeichen der Atrophie hervor. Beim Kaninchen gehen die Zellen der Drüsenröhrchen vorzugsweise durch einfache Atrophie zu Grunde; sie verringern sich im Volumen, verlieren die Zymogen-Körnchen, werden

1) Die Protocolle meiner Versuche kann ich wegen des grossen Raumes den sie einnehmen würden, hier nicht anfülren.

Archir f. pathol. Anat. Bd. 168. Hfft. 1. 
homogen und erhalten eine gewisse Aehnlichkeit mit den Epithelien der inneren Ausführungsgänge. Die Kerne der Drüsenröhrchen-Zellen nehmen eine unregelmässige Form an, schrumpfen ein und färben sich intensiver wie sonst (Pyknosis). Endlich schwinden die Zellen ganz, und und ihre Membrana propria fällt zusammen. Nebenkerne sah ich schon in den ersten Tagen nach der Operation nicht mehr; centroacinäre Zellen konnte ich vom 11. Tage an nicht mehr wahrnehmen und vermochte mir auch keine klare Vorstellung darüber zu machen, auf welche Weise diese ersteren Elemente schwanden. Die einzelnen Drüsenröhrchen und Läppchen verfallen nicht gleichzeitig der Atrophie, sie verschwinden in einigen Läppchen die Drüsenröhrchen vollständig schon am 15. Tage, in anderen Läppchen dagegen bleiben sie anscheinend bis zum 30. Tage erhalten. Eine klare Unterscheidung der Drüsenröhrchen von den Ausführungsgängen war in Versuchen von solcher Dauer infolge der schon erwähnten Veränderungen im Charakter der Drüsenzellen nicht möglich. Ausser den Erscheinungen einer Atrophie in Form einer einfachen Atrophie oder seltener in Form einer Vacuolisation und körnigen Zerfalls, konnte man in den ersten Tagen in den Zellen der Drüsenröhrchen auch eine Vermehrung durch indirecte Theilung wahrnehmen.

Vom 3. Tage an kann man in den Ausführungsgängen die Erscheinungen einer Vermehrung der Epithelzellen beobachten, die Zellen werden grösser und theilen sich. Am zahlreichsten trifft man Mitosen in den feinkalibrigen Ausführungsgängen, weniger zahlreich in den mittelweiten und sehr selten in den weiten Gängen. Ausserdem bemerkt man zwischen dem 4. bis 15. Tage nach der Operation im Epithel der grosskalibrigen Ausführungsgänge beim Kaninchen eine schleimige Degeneration vereinzelter Zellen (Becherzellen) und entsprechende Schleimklümpchen im Inhalt der Gänge. Die Wandung der Ausführungsgänge ist theils durch neugebildetes Bindegewebe, theils durch Aneinanderrücken des. Bindegewebs-Stroma der Drüse verdickt. Vom 30. Tage an beginnt die Atrophie der feineren Ausführungsgänge; sie fallen zusammen, ihr Epithel geht durch einfache Atrophie mit Pyknosis der Kerne zu Grunde, die Zabl der feineren und mittelweiten Gänge verringert sich allmählich, 
bis zuletzt, 400 Tage nach der Operation, nur der Hauptgang mit verhältnissmässig dünner Wandung erhalten bleibt, in dessen Wandung ich, nebenbei bemerkt, 200 Tage nach der Operation Kalk-Ablagerungen beobachtet habe. Im Inhalte der Ausführungswege wurden in der ersten Zeit feine Körnchen beobachtet, die auch der Färbung nach den Zymogen-Körnchen ähnelten, ausserdem die schon erwähnten Schleimklümpchen und hin und wieder auch Leukocyten.

Die Langerhans'schen Inseln bleiben beim Kaninchen bis zum 400. Tage nach der Unterbindung des Ausführungsganges erhalten, d. h. bis zu einer Zeit, wo alle entzündlichen Processe in der Drüse längst ihr Ende erreicht haben, das neugebildete Bindegewebe aber theils atrophisch ist, theils den Charakter des Fettgewebes angenommen hat, und man also für das weitere Bestehen der Inseln keinerlei Befürchtungen zu hegen braucht. In einem früheren Stadium, zwischen dem 30. und 120. Tage nach der Operation, waren die Ernährungs-Bedingungen und die Druck-Bedingungen von Seiten des benachbarten Bindegewebes nicht so günstige, und ein Theil der Inseln, ürigens nur ein sehr geringer, ging völlig zu Grunde, während vereinzelte Inseln sich nur im Volumen verringerten. Dabei war der Grad der Atrophie der Inseln bei den einzelnen Individuen ein verschiedener, trotz der sonst gleichen Bedingungen. Die gleichen Verhältnisse beoabachtete ich auch an Hunden; bei einem Hunde wurde 15 Tage nach der Operation eine, wenn auch nicht intensive, fettige Degeneration der Inseln constatirt. Ein unter gleichen Bedingungen an einem anderen Hunde angestellter Controllversuch ergab nichts Derartiges. Die Facta führen mich zu der Annahme, dass die Inseln bei verschiedenen Vertretern einer und derselben Art gegen schädlichen Einflüssen über einen verschiedenen Grad von Resistenz-Fähigkeit. verfügen. In den Inseln blieben auch beide Arten von Zellen erhalten, nur änderten sich in den im Volumen verkleinerteo Inseln das Verhältniss der beiden Arten von Zellen zu einander in dem Sinne, dass die Zahl der grossen Zellen relativ grösser wurde. Von der Drüse blieben also, wenn wir die grossen Ausführungsgänge nicht mitzählen, nur die Inseln erhalten, und das genügte, um das Entstehen eines Diabetes zu verhindern. Nur 
eine Total-Exstirpation der Drüse, d. h. eine Entfernung ihres Verdauungsapparates und der Inseln führt zur Entstehung dieser Krankheit. Wir erhalten also als Resultat eine vollständige Bestätigung der ersthin ausgesprochenen Hypothese, dass nehmlich die Inseln die Träger einer zweiten besonderen, in Beziehung zum Umsatz der Kohlenhydrate stehenden Function der Drüse sind und selbständige Gebilde darstellen, die mit dem Verdauungs-Apparat der Drüse und seinen Ausführungsgängen in keinerlei Beziehung stehen.

In dem die Ausführungsgänge umgebenden interlobulären Bindegewebe der Drüse wächst beim Kaninchen allmählich die Zahl der Zellen, anfangs Dank einer Ansammlung von WanderElementen und Volumen-Vergrösserung der sesshaften Zellen, dann aber vom 5. bis 6. Tage an auch durch Vermehrung dieser letzteren Elemente, die am 8. Tage ihr Maximum erreicht. Um diese Zeit beobachtet man sehr viele Mastzellen and epithelioide Zellen. Zwischen den einzelnen Drüsenröhrchen ist die Neubildung von Bindegewebe eine ganz geringe und eine Zunahme desselben nur scheinbar und relativ. Im Allgemeinen gehen die Erscheinungen einer productiven Entzündung nach 30-45 Tagen zurück, und von diesem Zeitpunkte an findet man nur stellenweise einzelne, kleine, kleinzellige Infiltrations-Heerde, die Menge des Bindegewebes aber beginnt allmählich abzunehmen, theilweise wird es durch Fettgewebe ersetzt. Was das elastische Gewebe anbetrifft, so ist eine recht bedeutende Neubildung desselben um die Ausführungsgänge herum, rings um die Inseln und an den Stellen, wo die Drüsenläppchen geschwunden sind, zu constatiren. Anfangs beobachtet man in der atrophirenden Drüse viele Blutgefässe und markhaltige Nervenstränge, doch beginnt die Zahl der ersteren nach 20 Tagen, die der letzteren nach 60-75 Tagen abzunehmen. Die Zahl der Nervenknoten und die Zahl der Zellen in den restirenden Knoten wird gleichfalls kleiner. Einzelne Nervenknoten und Nervenstränge bleiben jedoch bis ganz zuletzt unverändert. Möglicherweise regeln diese erhaltenen Nervenknoten die Function der Inseln, während die atrophirten Nervenknoten in der gleichen Beziehung zur Verdauungsthätigkeit der Drüse standen. Von Popelsky sind in letzter Zeit meine Ansichten durch physiologische Experimente 
bestätigt worden, während dieser Autor früher eine Localisation des Centrums im Magen, in der Nähe des Pylorus, annahm. Beim Hunde geht die Atrophie etwas langsamer vor sich, so dass in einigen Läppchen die Drüsenröhrchen bis zum 50. Tage erhalten bleiben; die Zellen der peripherisch gelegenen DrüsenRöhrchen gehen durch einfache Atrophie zu Grunde, während die der centralen Theile eine fettige Degeneration mit Chromatolisis der Kerne erleiden. Der Process der Neubildung in den Ausführungsgängen ist beim Hunde schwach ausgeprägt, dagegen geht aber die Neubildung von interlobulärem Bindegewebe rascher vor sich, wie beim Kaninchen. Die Zahl der Capillargefässe nimmt auch beim Hunde mit dem Fortschritte der Atrophie ab, was aber die Nerven und die Nervenknoten anbelangt, so konnte ich, vielleicht in Folge der relativ kurzen Beobachtuugs-Dauer von 50 . Tagen nach der Unterbindung nichts Besonderes constatiren.

Bei der Katze geht der Process etwas rascher vor sich, wie beim Hunde, und ergreift die Läppchen in mehr diffuser Weise. Die Zellen der Drüsenröhrchen erleiden nur eine geringe fettige Degeneration und gehen vorzugsweise durch eine solche Atrophie zu Grunde; eine Neubildung von feinkalibrigen Ausführungsgängen ist stärker ausgesprochen, als beim Hunde, doch schwächer, wie beim Kaninchen. Die Bindegewebs-Neubildung dagegen ist stärker, als beim Kaninchen, doch schwächer, als beim Hunde. Was die Gefässe und Nerven anbelangt, so konnte ich nichts Besonderes nachweisen, da bei der Katze die Durchgängigkeit des Ausführungsganges sich bald wieder herstellt und die Drüse regenerirt. Nur scheinen bei Beginn der Atrophie die Vater-Pacini'schen Körper zu Grunde zu gehen, um auch bei der späteren Regeneration der Drüse nicht wieder zu erscheinen.

Die Langerhans'schen Inseln überlebten beim Hunde, der Katze und dem Kaninchen alle Elemente der Drüse und blieben heil und unverändert, trotzdem die Druckwirkungen von Seiten der benachbarten Ausfübrungsgänge uud DrüsenRöhrchen auf die Inseln nur wenig geringer sind, dagegen der Druck von Seiten des Bindegewebes sowohl auf die Inseln, wie auf die übrigen Elemente der Drüse ein gleicher ist. Die 
Circulations-Bedingungen müssen sich, wenn sie sich ändern, in gleicher Weise ändern, da die Inseln kein eigenes GefässSystem haben. Man muss daher annehmen, dass irgend welche anderen Bindingungen vorhanden sind, Dank denen, trotz des vollständigen Unterganges des übrigen Parenchyms die Inseln erhalten bleiben. Als solche Bedingungen liessen sich nur trophische Einflüsse anführen oder aber eine gewisse angeborene Widerstandsfähigkeit der Inselzellen schädlichen Einflüssen gegenüber. Die Inseln vermögen auch nach der Unterbindung des Ausführungsganges der Drüse ungehindert ihre Function zu erfüllen, wobei sie in normaler Weise dazu angeregt werden und ihre Erhaltung für den Organismus unzweifelhaft von grosser Wichtigkeit ist.

Ganz anders verhält sich die Sache mit dem VerdauungsApparat der Drüse und zwar auch verscbieden für die einzelnen Theile dieses Apparates. Nach der Unterbindung dieses Ausführungsganges kann der Verdauungs-Apparat der Drüse dem Organismus keinerlei Nutzen bringen und kann erst nach der Wiederherstellung des Ganges wieder nutzbringend wirken. In der That sehen wir, dass der Organismus diese Bedingungen gleichsam zu verstehen scheint, wir sahen, dass die DrüsenRöhrchen atrophirten und der Organismus bestrebt war, durch Neubildung von Ausführungsgängen die Durchgängigkeit der Ausführungswege wieder herzustellen, was ihm auch gelang, wenu nicht Maasregeln getroffen waren, um diese Thätigkeit zu verhindern oder durch Resection grosser Stücke des Ausführungsganges der Regenerations-Fähigkeit des Organismus Grenzen gezogen waren. Stellt sich aber die Durchgängigkeit des Ausführungsganges wieder her, so erfolgt sofort und ungemein rasch, beim Hunde schon im Verlauf von 10 Tagen, eine Wiederherstellung des Verdauungs-Apparates der Drïse von Seiten der erhatten gebliebenen Drüsen-Röhrchen und Ausführungsgänge. Die Drüse stellt sich dabei in ihrem früheren Umfange und Form wieder her und unterscheidet sich mikroskopisch von einer normalen Drüse nur durch eine weniger gleichmässige Grösse der Läppchen und eine häufigere Anordnung der Inseln in den pheripherischen Theilen der Drüsen. Bei dieser Regeneration der Drüse erleidet das neugebildete feste Bindegewebe, das beim 
Hunde in bedeutender Menge vorhanden ist, Veränderungen regressiver Natur, die vielleicht bedingt sind durch den Druck von Seiten der regenerirenden Elemente der Drüse.

Dieses Factum steht scheinbar im Widerspruch zu den feststehenden Anschanungen über die Bedeutung des Druckes von Seiten des in den Organen neugebildeten fibrösen Bindegewebes auf das Parenchym der betreffenden Organe. Wenn aber die Versuche einer Wiederherstellung des Ausführungsganges längere Zeit resultatlos geblieben und die secretorischen Drüsen-Elemente zu Grunde gegangen sind, so wird eine Neubildong von Ausführungsgängen und die Existenz dieser Gänge für den Organismus nutzlos, und in der That sehen wir eine allmähliche Verringerung der Zahl der Gänge und eine Kalk-Incrustation der Wandung der restirenden grösseren Ausführungsgänge.

Bei der Durchsicht der Literatur finden wir die verschiedensten Anschauungen über die Structur und Bedeutung der Inseln. Langerhaus, der sie zuerst beschrieben hat, hält sie für nervöse Elemente, Saviotti sprach im selben Jahre die Ansicht aus, dass die Inseln zum System der Ausführungswege gehören. Renaut und nach ihm Monret hielten die Inseln für Lymphfollikel, Gibbes für Embryonalreste, Jarotsky sieht in den Inseln Elemente epithelialen Charakters, die seiner Ansicht nach Stoffe absondern, die für Bereitung des Fermentes durch die Drüsenröhrchen nötig sind. Kühne und Lea, die eine vortreffliche Beschreibung der Inseln geliefert haben, enthalten sich jeglicher Hypothese über ihre Funetion, desgleichen auch Podw yssotzky. v. Ebner (Köllikers Lehrbuch) hält die Inseln für selbständige Gebilde und stellt die richtige Hypothese auf, dass sie eine Rolle beim Umsatz der Kohlenhydrate spielen.

Als ich meine Arbeit schon begonnen hatte, erschien die Mittheilung von Diamare, der gleicher Ansicht ist und die Absicht ausspricht, sich später mit der Untersuchung des Pankreas nach Unterbindung des Ausführungsganges zu beschäftigen. Endlich hält eine ganze Gruppe von Autoren, angefangen von Lew aschew, die Inseln auf Grund der Vergrösserung ihrer Zahl bei Thieren während der Verdaung, nach PiloearpinInjectionen und nach längerem Hungern (Statkewitsch) und auf Grund der Uebergangsformen für abgearbeitete, erschöpfte Drüsen- 
Elemente (Dogiel, Pischinger, Laguesse, Pugnat, Mankowsky, Statkewitsch, Tschassownikow). Aber Uebergangsformen lassen sich ja beibeliebigen Zellformen finden, so hat ja seiner Zeit selbst R. Virchow auf Grund dieser alleinigen Methode die Krebszellen von den Leukocyten abgeleitet. Ferner erscheint zwar die Zahl der Inseln während der Thätigkeit der Drüse thatsächlich vergrössert, doch nur in geringem Grade und abhängig von einer Volumen-Verminderung des Verdauungs-A pparates der Drüse. In einem meiner Versuche entnahm ich per Laparatomiam einem Hunde ein kleines Stück der Bauchspeicheldrüse; nach Verheilung der Wunde unterwarf ich den Hund einer verstärkten Pilocarpin-Behandlung und tödtete ihn. Die Zahl der Inseln erwies sich nach der Pilocarpin-Behandlung nur in ganz geringem Maasse vergrössert. Ferner stellte ich auch zur Nachprüfung der Versuche von Statkewitsch 4 Versuche mit Hungern und ausschliesslicher Ernährung durch Kohlenhydrate an Thieren an. Ich setzte dabei voraus, dass die Inseln als thätige Organe geringere Veränderungen erleiden müssten und in Folge einer Atrophie des unthätigen Verdauungs-Apparates der Drüse in relativ grösserer Ánzahl gefunden werden müssten. Die mikroskopische Untersuchung der Drüsen bei diesen Versuchen bestätigte meine Annahme.

Stschastny fand bei der Untersuchung der Bauchspeicheldrüse eines nach 35 tägigem Hungern gestorbenen Menschen (es handelte sich um einen Geisteskranken) die Inseln, im Gegensatz zum Verdaungs-Apparat der Drïse, sehr gut erhalten; dasselbe fand auch Jarotzky.

Die Literatur über die Unterbindung des Ausführungsganges des Pankreas ist nicht sehr umfangreich. Die ersten Forscber, die diese Operation machten, sind meines Wissens Cl. Bernard (1856) und nach ihm Munk und Klebs; eine mikroskopische Untersuchung erwähnen diese Autoren nicht. In den weiteren Arbeiten über diese Frage von J. P. Pawlow (nicht S. Pawlow, wie in Pflüger's Archiv Bd. 16 gedruckt ist), Ribbert, Remy und Showe, Arnozan et Vaillard, Senn, Pawlow und Smirnow werden die Inseln nicht erwähnt, aber aus der Beschreibung von Ribbert und Arnozan geht hervor, dass sie ein Erhaltenbleiben der Inseln beobachtet haben. Treuberg spricht von 
einem Erhaltenbleiben der Inseln in einem Versuche, misst aber diesem Factum keine weitere Bedeutung zu.

Nach meiner vorläufigen Mittheilung erschienen die Arbeiten von W. Schultze aus dem Laboratorium von Hertwig und von Mankowsky aus dem Laboratorium von Podwyssotzky. Der erste dieser Autoren kam unabhängig von mir, was er auch bestätigt, zu demselben Schluss; der zweite kam in seiner sonderbaren Arbeit zu ganz entgegengesetzten Resultaten: er giebt an, dass die Langerhans'schen Inseln nach der Unterbindung des Ausführungsganges zu Grunde gehen und zweifellos zu den Ausführungswegen der Bauchspeicheldrüse in Beziehung stehen.

Endlich stellte ich poch 3 Versuche an Kaninchen an, denen ich vor der Unterbindung des Ausführungsganges der Drüse Oleum provinciale in denselben einführte. Hédon erhielt bei dieser Modification des Experiments manchmal bei Kaninchen vorübergehende Glykosurie. Zwei meiner Kaninchen überstanden die Operation sehr gut; doch hatten sie keine Glykosurie. Beide wurden nach 30, bezw. 60 Tagen getödtet, und ihre Bauchspeicheldrüsen wiesen dieselben Veränderungen auf, die wir bei der einfachen Unterbindung des Ausführungsganges beobachtet hatten. Ein Kaninchen ging 2 Tage nach der Operation ein; bei der Section erwies sich partielle Nekrose des Pankreas, und zwar zeigte das interlobuläre und das die Drüse umgebende Fettgewebe die Anzeichen einer Fettgewebs-Nekrose; mikroskopisch liess sich ferner eine Nekrose vieler Drüsenläppchen und Inseln nachweisen. Man kann daher annehmen, dass in den Versuchen von Hédon nur ein Theil der Inseln nekrotisch wurde, die übrigen aber nur für eine gewisse Zeit in ungünstige Verhältnisse versetzt wurden, weshalb auch bei den Thieren eine vorübergehende Glykosurie sich einstellte. Bei diesem Versuch stellte das $0 e l$ in dem Ausfijhrungsgange eine weissliche breiartige Masse dar. Die Beobachtungen über die FettgewebsNekrose bringen mich auf den Gedanken, dass zum Zustandekommen dieser Erscheinung das Vorhandensein zweier Factoren nöthig ist, - die Anwesenheit von Ferment, welches das Fett spaltet, und eine verringerte Lebensfähigkeit der Fettgewebszellen. Besonders deutlich trat dies zu Tage in den Versuchen mit Durchschneidung des Ausführungsganges, - eine Nekrose war 
nur um die Ligatur herum sichtbar. Eine derartige Hypothese wurde von Martynow ausgesprochen, und in letzter Zeit stellen Katz und Winkler, die eine genaue Literatur-Besprechung über diese Frage liefern, noch als zweites Moment eine Störung der Blut-Circulation hin. Eine solche Hypothese erklärt aber schon einige Facta, wie sie z. B. Hlava erhielt, nicht mehr.

\section{Versuche mit Transplantation der Drüse.}

Die Untersuchungen von Minkowsky, Gley, Thiroloix, Hédon zeigten, dass bei Transplantation eines Stückchen Pankreas mit gefässhaltiger Brücke unter die Haut und Exstirpation des übrigen Theiles der Drüse nach Anheilung des Drüsenstückchens kein Diabetes eintritt, trotz der Atrophie im transplantirten Drüsentheil. Nach der Entfernung dieses Drüsenstückehens zeigt sich aber bei den Thieren schon nach einigen Stunden Zucker im Harn.

Dabei erwies es sich, dass die Ausscheidung von PankreasSecret aus der Fistel des Ausführungsganges des transplantirten Drüsentheils vollständig aufhören kann. Auch eine umgekehrte Erscheinung wurde beobachtet (Thiroloix). Bei einem Thier treten sehr bald nach der Exstirpation des in der Bauchhöhle verbliebenen Theils der Drüse die Erscheinungen eines Diabetes auf, die Secretion aus dem transplantirten Stücke dauerte jedoch fort. Auf Grund dieser Thatsachen kamen die Forscher zu dem Schluss, dass die innere Secretion der Drüse unabhängig ist von ihrer äusseren Secretion, und dass zum normalen Verlauf des Umsatzes der Kohlenhydrate das Eintreten der Producte dieser inneren Secretion in das Blut genügt. Mouret unterwarf mehrere derartig transplantirte Pankreastheile einer mikroskopischen Untersuchung. Diese unter der Haut liegenden Drüsentheile functionirten in Bezug auf den Umsatz der Kohlehydrate vollkommen gut, und erst nach ibrer Exstirpation trat Diabetes auf. Die Langerhans'schen Inseln erwähnt Mouret bei dieser Untersuchung nicht, weist aber anf die Atrophie und Sklerose des Parenchyms hin. Weitere einigermaassen detailirte Untersuchungen dieser Art habe ich nicht finden können.

Ich machte 2 Versuche an Hunden mit Transplantation des freien verticalen Theiles des Pankreas unter die Bauchhaut 
Nach 30-40tägigem Bestehen hörte die Secretion aus der Fistel des Ausführungsganges des transplantirten Drüsentheiles auf. 50 und 130 Tage nach der Operation untersuchte ich die transplantirten Stücke. Im ersten Falle bestand es aus einzelnen erbsengrossen Knötchen. Diese Knötchen bestanden aus stark atrophischen Drüsenläppchen und verhältnissmässig gut erhaltenen Langerhans'schen Inseln. Dabei fanden sich viele Nervenknoten. Nach 130 Tagen fanden sich an Stelle des transplantirten Drüsenstückes sehr kleine, grau gefärbte Knötchen, bestehend aus Resten der Ausführungsgänge, einer geringen Anzahl von Inseln und veränderten Nervenknoten. Diese Versuche zeigen, dass bei einer Transplantation unter die Haut die Drüse in toto atrophiren kann, dabei aber die Langerhans'schen Inseln sich als die widerstandsfähigeren Elemente erweisen und auch das Eintreten eines Diabetes verhindern. Ein Exstirpation des intra-abdominellen Theiles der Drüse konnte ich nicht ausführen; nach dieser Operation bleiben die Inseln vielleicht darum erhalten, weil ihre Existenz für den Organismus eine conditio sine qua non bedeutet.

III. Versuche mit Hervorrufung verschiedener Thätigkeits-Stadien der Inseln.

Analog den bekannten Versuchen von R. Heidenhain am Verdauungs-Apparat der Drüse, versuchte ich das Thier bezüglich des Umsatzes der Kohlehydrate verschiedenen Bedingungen zu unterwerfen, um dabei das Protoplasma der Inselzellen auf Körnchen-Reichthum zu untersuchen. Ich setzte voraus, dass bei kurzem Hungern die Inselzellen sich einer relativen Ruhe erfreuen würden, bei verstärkter Fütterung mit Kohlehydraten dagegen eine intensive Thätigkeit derselben zu erwarten" wäre. Ferner exstirpirte ich, um einen derartigen Zustand erhöhter Thätigkeit hervorzurufen, einem Hunde mehr als $\frac{2}{3}$ der Drüse und unterwarf darauf das Thier einer verstärkten Fütterung mit Kohlehydraten. Die grösste Anzahl Körnchen im Protoplasma der Inselzellen wurde bei 2-3tägigem Hungern beobachtet, eine etwas geringere Menge Körnchen bei normalen ErnährungsBedingungen und bei Hungern plus intravenöser Injection von Glykose-Lösung (die Hunde wurden 5 Stunden nach der Injection 
getödtet). Eine noch geringere Anzah! Körnchen fand ich bei verstärkter Fütterung mit Kohlehydraten, verbunden mit intravenöser Zucker-Injection. Ferner wurde noch ein Hund, dem $\frac{2}{3}$ der Drüse exstirpirt waren, und der diese Operation gut überstanden hatte und sich vollkommen erholt hatte, einer verstärkten Fütterung mit Kohlehydraten, verbunden mit intravenöser Zucker-Infusion, unterworfen. In diesem Falle wurde eine deutlich wahmehmbare Volumen-Verkleinerung der Inselzellen beobachtet, wobei das Protoplasma derselben fast gar keine fucbsinophilen Köruchen enthieit, dagegen sich Fetttröpfehen in ihm fanden (Taf. IV, Fig. 6 u. 7). Diese Versuche zeigten, dass in den Zellen der Langerhans'schen Inseln irgend eive Substanz in Form von Körnchen, die den Zymogenkörnchen ähneln, erzengt wird. Die grössten dieser Körnchen liegen an der Peripherie der Zelle. Die Zahl dieser Körnchen, die bei kurzem Hungern am grössten 'ist, verringert sich bedeutend, wenn an den Organismus die Anforderung gestellt wird, auf diese oder jene Weise ein gewisses Quantum von Kohlehydraten auszunutzen, d. h. sie entweder in Form von Glycogen in den Geweben abzulagern, oder in andere Substanzen, z. B. Fett, zu verwandeln, oder endlich sie einfach zu verbrennen. Es ist ja bekannt, dass bei fehlender Function der Bauchspeicheldrüse bei Diabetes der Organismus die Fähigkeit verliert, den Zucker der Nahrung zu assimiliren, ihn im Körper zurüekzuhalten und zu verbrauchen.

\section{Embryologie.}

Ich versuchte, meine Hypothese von der Bedentung und Function der Inseln auch auf embryologischem Wege auf ihre Richtigkeit hin zu prüfen.

Allgemein bekannt ist ja das Factum, dass beim Embryo und bei jungen Individuen im Anfange des extra-uterinen Lebens die Blutdrüsen, d. h. die Drüsen mit innerer Secretion, verhältnissmässig stärker entwickelt sind, als die Drüsen mit äusserer Seeretion. Daher kann man erwarten, dass die Langerhansschen Inseln, wenn sie thatsächlich Organe mit innerer Secretion vorstellen, beim Embryo gut entwickelt sein werden, ja in einer gewissen Periode sogar besser, als der Verdauungs-Apparat der Drüse. 
Mir stand folgendes Material zur Verfügung: die Bauchspeicheldrüse eines menschlichen Foetus von $31 \mathrm{~cm}$ Länge, eines neugeborenen und eines 10 Monate alten Kindes, die Drüsen eines neugeborenen und eines 3 Wochen alten Kaninchens, eines 5 Tage alten Meerschweinchens und die Drüsen von 5 Kälbern.

Beim menschlichen Foetus treten die Inseln inmitten des übrigen Parenchyms deutlich hervor in Form von rundlichen Häufchen polygonaler Zellen; der Flächen-Inhalt der Inseln im Verhältniss zum übrigen Parenchym beträgt auf den Schnitten 1 oder $\frac{3}{2}$ (Taf. IV, Fig. 8). Beim neugeborenen Kinde sind die Inseln etwas grösser (bis $0,4 \mathrm{~mm}$ im Durchmesser) und fioden sich auf den Schnitten in geringerer Anzahl; beim 10 Monate alten Kinde sind die Inseln etwas kleiner, als beim Erwachsenen, und ihre Zahl auf dem Schnitt, Dank einer stärkeren Entwicklung des Verdaungs-Apparates, eine noch geringere, als beim Neugeborenen, doch immerhin auch hier grösser, als beim Erwachsenen.

Der Verdauungs-Apparat der Drüse beim 6 Monate alten menschlichen Foetus ist relativ schwach entwickelt und besteht aus einigen gewundenen Drüsenröhrchen und Ausführungsgängen. Eine Differenzirung in Läppchen fehlt, das fibrilläre Bindegewebsstroma ist stark entwickelt.

Beim neugeborenen Kaninchen and besonders bei dem 5 Tage alten Meerschweinchen ist der Verdaungs-Apparat der Drüse schon recht gut entwickelt. Bei dem ersteren ist die Abgrenzung der Inseln von den Drüsenröhrchen nicht scharf, die Zellgrenzen undeutlich; beim Meerschweinchen dagegen sind die Inseln scharf abgegrenzt, ihr Durchmesser etwas kleiner, als beim ausgewachsenen Thier; dabei ist die Zahl der grossen, körnigen Zellen relativ grösser. Beim Kaninchen und Meerschweinchen findet man nicht selten Kerntheilungs-Figuren in den Inselzellen (Taf. IV Fíg. 9). In den Präparaten der Drüse eines 3 Wochen aiten Kaninchens traten die InseIn nach der Iujection der Blutgefässe mit Carmin-Gelatinemasse sehr deutlich hervor und waren zahlreiches zu finden, als beim ausgewachsenen Kaninchen; deutlich sichtbar war dabei auch die Anordnung der Zellen za Reihen und Colonnen von der Breite einer Zelle; alle Zwischenräume zwischen den Colounen waren mit Injections-Masse ausgefüllt (Taf. IV, Fig. 10). Bei den Kälbern finden sich die Inseln in 
der Drüse dichter bei einander gelagert, als beim Rinde, doch von fast gleicher Grösse und aus Protoplasma-reichen durchsichtigen Zellen bestehend.

Schon die Thatsache, dass man bei einem 6 monatlichen menschlichen Foetus die Inseln findet, weist darauf hin, dass das keine abgearbeiteten erschöpften Zellen der Drüsenröhrchen sein können, da ja zu dieser Zeit natürlich von einer bis zu solcher Erschöpfung führenden secretorischen Thätigkeit der Drüse nicht die Rede sein kann. Die Anwesenheit von Mitosen und die Grösse der Inseln beweist noch mehr, dass es Gebilde sui generis sind, Gebilde mit selbständigem Wachsthum, nicht aber abgearbeitete Drüsenzellen, "todte Punkte", die durch fettige Degeneration zu Grunde gehen (Dogiel). Die absolute Zahl der Inseln in jeder einzelnen Drüse unterliegt, wie es diese und frühere Untersuchungen zeigen, im Verlaufe des ganzen Lebens des Organismus keiner Aenderung im Sinne einer Vermehrung. Dagegen kann die Zahl der Inseln durch pathologische Bedingungen sehr wohl verringert werden, ja dieselben können gänzlich schwinden.

Die ersten Forschungen in dieser Richtung stellte, soweit mir bekannt ist, Podwyssotzky an, der aber bei neugeborenen Katzen keine Inseln in der Bauchspeicheldrüse finden konnte. Bizzozero und Vassale, Diamare fanden die Inseln bei Embryonen und sahen sie daher für selbständige Gebilde an. Die genaueste Untersuchung über die Structur der Bauchspeicheldrüse des Embryo ist wohl die von Laguesse; er fand die Inseln bei einem Schaf-Embryo von $18 \mathrm{~mm}$ Länge; nach Laguesse können sie aus primären und secundären DrüsenRöhrchen des Pankreas entstehen, erleiden aber mit der Zeit eine Rückbildung. Späterhin gab Laguesse zu, dass in gewissen Fällen die Inseln constante Gebilde vorstellen. Was die Function der Inseln anbelangt, so bringt auch Laguesse sie in Verbindung mit einer inneren Secretion. Gartier fand die Inseln schon bei einem 3 monatlichen menschlichen Embryo recht gut entwickelt, über ibre Function erwähnt er jedoch Nichts.

B. Pathologische Anatomie.

Dieser Theil meiner Arbeit enthält die Untersuchungen der 
Bauchspeicheldrüse bei verschiedenen Erkrankungen, die zur Atrophie des Parenchyms der Drüse führen, ausser Diabetes, und zweitens die Untersuchungen der Bauchspeicheldrüse von Diabetikern. Ich rechnete darauf, im ersten Falle die Inseln erhalten zu finden, im zweiten Falle dagegen, besonders bei schwerem Diabetes, bedeutende pathologische Veränderungen der Inseln oder aber eine beträchtliche Verringerung ihrer Zahl constatiren zu können. Diese Untersuchung soll eine klinische Prüfung des im experimentellen Theile meiner Arbeit aufgestellten Satzes sein, dass die Langerhans'schen Inseln das morphologische Substrat der inneren Secretion der Bauchspeicheldrüse darstellen, der Secretion, durch die ein normaler Verlauf des Umsatzes der Kohlehydrate im Organismus bedingt wird.

Dieser 'Theil meiner Arbeit zerfällt in zwei Abschnitte. 1. Die Untersuchung der veränderten Drüsen von NichtDiabetischen. 2. Die Untersuchung der Drüsen von Diabetischen.

I.

Mir standen 17 Fälle von Sklerose der Drüse mit Atrophie und 1 Fall von Lipomatosis zur Verfügung. Unter 6 Fällen war eine schwach ausgeprägte Sklerose, d. h. die Bindegewebs-Neubildung war nur längs der Gefässe und Ausführungsgänge zu constatiren, und auch hier war sie in einigen Fällen sehr gering, und die Erscheinungen der Atrophie praevalirten. Die Drüsenläppchen waren im Volumen verkleinert, die Grösse der Langerhans'schen Inseln unverändert; dieselben waren auf den Schnitten häufiger zu treffen, waren manchmal nur von einer geringe Anzahl von Drüsenröhrchen umgeben, bisweilen lagen sie ganz isolirt inmitten des Bindegewebes. In einem Falle war eine Neubildung von Bindegewebe nur an der Peripherie der Inseln und in den Inseln selbst nur um die Capillargefässe herum zu finden; manchmal nahm dabei das Bindegewebe altes fibrilläres Bindegewebe -, auf dem Schnitt fast die Hälfte einer Insel ein. Das Protoplasma der Inselzellen zeigte die Erscheinungen starker fettiger Degeneration, die stärker ausgesprochen war, als im Verdauungs-Apparat der Drüse; der Kranke hatte in diesem Falle ein Lymphosarcom des hinteren 
Mediastinalraumes. In 3 Fällen wurde eine Sklerose mittleren Grades beobachtet, davon in einem Falle hervorgerufen durch Steine aus kohlensaurem Kalk in den Ausführungsgängen. Die Neubildung von Bindegewebe war vorzugsweise auf die Ausführungsgänge besehränkt, das Gewebe selbst trug den Charakter. von festem Narbengewebe mit zahlreichen, spindelförmigen Zellen und vereinzelten kleinzelligen Infiltrations-Heerden. Die Läppchen waren stellenweise durch dieses neugebildete Gewebe scharf von einander abgegrenzt, einzelne Läppchen bis auf die Ausführungsgänge und die Langerhans'schen Inseln zu Grunde gegangen. In den erhalten gebliebenen Läppchen waren die Zellen im Volumen verkleinert. Die gut erhaltenen Inseln lagen stellenweise isolirt im fibrösen Bindegewebe oder in einem kleinzelligen Infiltrations-Heerde (vergl. Taf. IV, Fig. 11 und 12). In 2 Fällen starker Sklerose, von denen in einem Falle dieselbe auch durch Steine in den Ausführungsgängen verursacht war, war die Bauchspeicheldrüse fast gauz in festes fibröses Geweve und Fettgewebe verwandelt, und nur vereinzelt fanden sich Reste des Parenchyms in Form kleiner Knötchen von der Grösse eines Hanfkorns. Mikroskopisch liessen sich inmitten des fibrösen Bindegewebes und Fettgewebes Gruppen von unveränderten Langerhans'schen Inseln nachweisen, und hier und da auch geringe Reste von mehr oder weniger veränderten DrüsenRöhrchen und Ausführungsgängen. In dem Falle von Lipomatosis fand sich an Stelle der Drüse eine Fettmasse von beträchtlichem Umfange, die von kleinen, Hanfkorn grossen, weisslichen Knötchen durchsetzt war. Diese letzteren erwiesen sich unter dem Mikroskop als Reste der Drüsenröhrchen und der Langerbansschen Inseln. Von den Inseln waren verhältnissmässig wenige erhalten, stellenweise lagen sie isolirt.

Ferner untersuchte ich die Drüse bei geringer Sklerose derselben in einem Falle von erworbener Syphilis und in 4 Fällen von heriditärer Syphilis bei Neugeborenen, die spätestens am 8. Tage nach der Geburt gestorben waren. In diesen Fällen liessen sich dieselben Veränderungen constatiren, die in Fällen schwacher Sklerose der Drüse bei Nicht-Syphilitikern beobachtet wurden. Endlich war in einem Falle heriditärer Syphilis bei einem neugeborenen Kinde die Bauchspeicheldrüse vergrössert und von derber 


\section{3}

Consistenz und erwies sich unter dem Mikroskop als fast ganz aus fibrösem Gewebe bestehend mit recht vielen spindelförmigen Zellen, wobei noch die Fibrillen hyalin degenerirt waren und sich mit Haematoxylin färben liessen - Kalk-Infiltration. Um die Gefässe herum fanden sich concentrische Auflagerungen von feinen Fibrillen, die auf dem Schnitt den Vater-Pacini'schen Körperchen ähnelten. Vom Parenchym waren stellenweise nur die Ausführungsgänge erhalten und hier und da vereinzelte Drüsenröhrchen. Die Langerhans'schen Inseln waren überall gut orhalten, lagen stellenweise isolirt, und die in ihnen verlaufenden Gefässe wiesen keinerlei sichtbare Veränderungen auf.

In zwei von mir untersuchten Fällen einer haemorrhagischen Pankeatitis fand sich eine Blut-Infiltration und Nekrose aller Gewebselemente, sowohl der Inseln, wie der Drüsenröhrchen.

In einem von mir untersuchten Falle von Pseudoleukaemie erwies sich die Bauchspeicheldrüse vollkommen normal, während man doch bei einer Vergrösserung äller Körperdrüsen nach der Hypothese von Renaut und Mouret eine Volumen-Vergrösserung der Inseln hätte erwarten können:

Diese Reihe von Untersuchungen bestätigte die anfängliche Voraussetzung, dass bei atrophischen Veränderungen der Bauchspeicheldrüse bei Nicht-Diabetikern die Inseln erhalten bleiben müssen. Ferner wurde dadurch auch ein anderer, im experimentellen Theile der Arbeit gezogener Schluss bestätigt, dass nehmlich die Langerhans'schen Inseln auch beim normalen, richtiger gesagt, beim nicht an. Diabetes leidenden Menschen sich als äusserst widerstandsfähige Elemente verschiedenen schädlichen Einflüssen gegenüber erweisen, jedenfalls widerstandsfähiger sind, als der VerdaungsApparat der Bauchspeicheldrüse.

Wenn man diesen Satz auch in Bezug auf die längs den Ausführungsgängen verlaufenden und infolge dessen dem Verdauungs-Apparat der Drüse mehr Schaden zufügenden, entzündlichen Vorgänge anfechten könnte, so kann man dies bei syphilitischen Erkrankungen der. Drüse nicht thun. Hier circulirt das schädliche Agens - das syphilitische Gift in den Blutbahnen, in ihnen Veränderungen hervorrufend, wirkt also 
in gleicher Weise auf alle Gewebselemente. Der Unterschied in der Reaction von Seiten dieser Elemente lässt sich also nur durch ihre physiologische Rolle und ihre relative Widerstandsfähigkeit erklären. Endlich haben diese Untersuchungen, ebenso wie die an Thieren angestellten Versuche gezeigt, dass die Widerstandsfähigkeit der Inseln grossen individuellen Schwankungen unterworfen ist. So hatten z. B. die Inseln in den Fällen starker Sklerose gar nicht gelitten, trotzdem in einem dieser Fälle auch noch Alkoholismus vorlag, in einem andern Falle von Sklerose dagegen concentrirte sich diese fast ausschliesslich auf die Inseln, deren Zellen eine recht beträchtliche fettige Degeneration erlitten hatten; auch in dem Falle von Lipomatosis hatten die Inseln recht stark gelitten. Alle diese Fälle zeigen auch, dass die Pathologie der Langerhans'schen Inseln eine andere sein muss, als die des Verdauungs-Apparates der Drüse.

Die zu diesem Theil der Arbeit gehörige Literatur ist recht umfangreich, doch finden sich über die Langerhans'schen Inseln fast gar keine Angaben. Um späteren Autoren die Mühe zu ersparen, war ich bestrebt, die Literatur möglichst vollständig zu sammeln. In einer ganzen Reihe von Untersuchungen finden wir nur eine makroskopische Beschreibung der Bauchspeicheldrüse (Ancelet, Charrin, Claessen, Drozda, Hecker, Lewin, Mraček, Müller, Virchow, Wegner, Lépine, Lanceraux, Chwostek). Bei der Beschreibung des mikroskopischen Bildes erwähnen folgende Autoren die Inseln nicht (Arnozan et Vaillard, Beck, Biedert, BirchHirschfeld, Bogdanow, Carnot, Huber, Melnikow, Mraček, Martynow, Oedmansson, Schmidt, Rosenthal, Thorel). Tilger erwähnt bei der Beschreibung der Sklerose der Drüse mit Cysten-Bildung in der Cauda die Inseln nicht, giebt aber in seiner Fig. 2 eine vortreffliche Abbildung derselben. Iwanowsky nennt in seiner Dissertation die Inseln nicht, beschreibt sie aber als Gruppen von Acini mit schwach ausgeprägtem acinösem Bau. Rodionow und Kasahara constatiren die Anwesenheit der Inseln sowohl in normalen, wie auch in veränderten Drüsen, in letzterem Falle in grösserer Anzahl; Schlesinger vermerkt das Factum, dass bei Sklerose 


\section{5}

der Drüse bei heriditärer Syphilis die Inseln, bei Schwund des übrigen Parenchyms, erhalten bleiben. Die ausführlichsten Untersuchungen sind die von Dieckhoff. Er fand in seinem VII., VIII., XII. und XIII. Falle die Inseln erhalten bei Untergang der übrigen Elemente der Drüsenläppchen, selbst bei Sklerosen haematogenen Ursprungs (Fall VIII). In neuester Zeit fand Lefas, dass bei Cirrhosen der Leber die Inseln gut erhalten bleiben. Von allen angeführten Autoren machte nur Dieckhoff auf diese Erscheinung aufmerksam, doch auf diesen Autor will ich noch später zurückkommen.

Ferner sah ich auch die Literatur über verschiedene Erkrankungen des Pankreas durch, Hinweise auf die Inseln fand ich aber nur bei Kudrewetzky. Wassiljew sah bei MagenKatarrhen bei Kindern cirrhotische Veränderungen der BauchSpeicheldrüse, fand aber die Inseln erhalten. Mandelstamm hat die Inseln augenscheinlich gesehen, erwähnt sie aber nicht. Bei anderen Autoren (Barlow, Chiari, Dallemagne, Fripp, Gerhardi, Guinard, Hahn, Junger, Kirillow, Klippel, Körte, Kostjurin, Pallier, Phedran, Rachmaninow, Seitz, Smith, Thiroloix) werden die Inseln nicht erwähnt. Bei den Beschreibungen des Pankreas-Carcinoms, die mir bekannt sind (Bardet, Pick, Leven, Litten, Parisot, Salomon, Tolot, Ziehl) und des Pankreas-Sarcoms (Chiari) werden die Inseln nicht erwähnt. Litten und Hansemann fanden in einigen Fällen bei Nicht-Diabetikern das ganze Drüsen-Parenchym durch carcinomatöse Neubildung ersetzt. Den Widerspruch zwischen den Sectionsbefunden und dem Experiment erklärt Hansemann dadurch, dass die Krebszelle als directer Nachkomme der Drüsenzelle bis zu einem gewissen Grade im Sinne einer positiven (inneren) Secretion fortfährt zu functioniren. Aber diese geistreiche Hypothese wäre nur zulässig bei Krebs, der von den Langerhans'schen Inseln ausgeht. Ausserdem liesse sich auch eine andere Erklärung finden. Die Inseln, die bei einem interstitiellen Processe inmitten des Bindegewebes allein nachbleiben, sehen nehmlich Carcinom-Heerden sehr ähnlich und unterscheiden sich von diesen nur durch die regelmässige Form der Zellen und durch das Fehlen von ReactionsErscheinungen, obgleich letzterés bei interstitiellen Processen 
nicht. immer richtig ist. Weder Litten, noch Hansemann erwähnen die Inseln, und es ist daher wohl möglich, dass sie dieselben von Carcinom-Heerden nicht unterscheiden.

II.

Mir standen die Drüsen von 15 Diabetikern und von 1 Falle von acuter Glykosurie zur Verfügung. In 6 von diesen 15 Fällen handelte es sich um eine schwere Form der Diabetes, - Diabète maigre -, in den übrigen Fällen gestatteten die knappen Daten keine Beurtheilung der Form der Krankheit.

In einem Falle handelte es sich allem Anschein nach um eine nervöse Form von Diabetes, die sich nach dem Ende zu verschärfte. Nur in einem dieser Fälle zeigte die Bauchspeicheldrüse die Erscheinungen einer Sklerose mit bedeutender Atrophie des Parenchyms; in der Mehrzahl der Fälle wies die Drüse makroskopisch nur die Anzeichen einer einfachen Atrophie auf (8: Fälle); in den übrigen 6 Fällen waren makroskopisch keine Veränderungen wahrzunehmen. Mikroskopisch waren in dem oben erwähnten Falle von Sklerose der Drüse von ihrem VerdauungsApparat nur wenige, im Volumen verkleinerte Läppchen vorhanden, in den übrigen Fällen zeigten sich die Erscheinungen einer mehr oder weniger stark ausgesprochenen Atrophie mit VolumenVerkleinerung der Läppchen und einzelner Zellen der Drüsenröhrchen. Was die Inseln anbelangt, so wiesen dieselben in 2 Fällen keinerlei sichtbare Veränderungen auf; der KrankheitsVerlauf war in diesen Fällen unbekannt. In 4 Fällen konnte ich die Inseln überhaupt nicht finden, in den übrigen 9 Fällen dagegen wurde eine bedeutende Verringerung ihrer Zahl bis :zu fast gänzlichem Fehlen beobachtet. In dem Falle von Diabetes nervösen Ursprunges war eine Verringerung der Zahl der Inseln und eine starke fettige Degeneration derselben wahrzunehmen. Ausserdem zeigten in 2 Fällen die erhalten gebliebenen Inseln die Anzeichen einer einfachen Atrophie mit Pyknosis der Kerne (Taf. V, Fig. 14) und Vacuolisation mit Chromatolysis der Kerne (Taf. V, Fig. 15).

In dem Falle acuter Glykosurie, die sich an eine GastroEnteritis anschloss, zeigte die Bauchspeicheldrüse parenchymatöse Veränderungen und partielle Nekrose. Die Schleimbaut des 
Duodenum war gleichfalls nekrotisirt. Die Inseln wiesen bedeutende Veränderungen auf, sogar in sonst gut erhaltenen Bezirken des Parenchyms; ihre Zellen waren klein, die Grenzen des Protoplasma wie ausgefressen, manchmal fehlte um den Kern herum das Protoplasma und einzelne Kerne färbten sich schlecht. Im Zwischengewebe fanden sich stellenweise um die Ausführungsgänge herum recht viel Leukocyten und Bakterien, Diplokokken und Stäbchen.

Fassen wir nun die erhaltenen Resultate zusammen, so müssen wir hinsichtlich der Inseln bei Diabetes zu einem dem früher aufgestellten Satze gerade entgegengesetzten Schlusse kommen, dass nehmlich die Inseln bei dieser Krankheit als die am wenigsten widerstandsfähigen Elemente erscheinen. In 13 von 15 Fällen warde eine Verringerung der Zahl der Inseln bis zu vollständigem Fehlen auf den Schnitten beobachtet. Die erhalten gebliebenen Inseln aber wiesen starke Veränderungen auf; viel stärkeren Grades, als die Veränderungen im VerdauungsApparat der Drüse. In diesem letzteren musste man ja bei dem krankhaft gesteigerten Appetit der Kranken eine gewisse Atrophie in Folge gesteigerter Thätigkeit der Drüse erwarten, und thatsächlich war in der Mehrzahl der Fälle diese Atrophie auch vorhanden. Ferner muss man, wenn man als Ursache des Diabetes den Schwund der Inseln ansieht, denselben nur bei der schwereren Form von Diabetes, - Diabète maigre -, suchen, die dem experimentell erzengten Diabetes analog ist und sich von diesem nur durch längeren Verlauf unterscheidet, weswegen man auch nicht immer ein vollkommenes Fehlen der Inseln in der Drüse zu finden erwarten darf. Bei nervösem und constitutionellem Diabetes (Fettleibige und Podagriker) haben wir keinen Grund, in den Inseln Veränderungen zu suchen; nur in den Fällen nervösér Diabetes, die zum Schluss das Bild eines wirklichen Diabète maigre mit raschem Verlauf zeigen, wären wir dazu berechtigt. Unter meinen Fällen war ein solcher Fall, und doch konnte ich auch Veränderungen in dèr Zahl der Inseln constatiren. In diesen Fällen kann der Untergang der Inseln eine secundäre Erscheinung sein, die dann zum Uebergange der Krankheit in Diabète maigre führt. Was den Mechanismus des nervösen Diabetes anbetrifft, so wird dabẹi möglicher Weise die 
Zucker-Ausscheidung im Harn durch eine functionelle Neurose der Inseln, als des Hauptregulators des Umsatzes der Kohlehydrate im Organismus, bedingt, wobei diese Neurose auch zum Untergange der Inseln selbst führt. Wir wissen ja, dass eine gewisse Widerstandsfähigkeit der Gewebe durch angeborene und erblich erworbene Eigenschaften bedingt wird, im späteren Leben aber auch trophische Einflüsse eine nicht geringe Rolle spielen. Die Facta, die wir in der Literatur finden, zeigen, dass Diabetes bei einigen Rassen (Juden) besonders häufig beobachtet wird, dass diese Krankheit ferner einige Gegenden bevorzugt (Malta), und dass endlich die Krankheit oder wenigstens eine Prädisposition zu ihr erblich ist, wie es ja die nicht seltenen Fälle von Diabetes bei mehreren Gliedern einer Familie zeigen.

Dass gerade eine Verminderung der Widerstandsfähigk eit der Zellen der Langerhans'schen Inseln die Ursache ihres Unterganges ist, das beweist bis zu einem gewissen Grade die Art und Weise ihres Unterganges, nehmlich durch einfache Atrophie der Zellen.

Die Inseln verschwanden in allen Fällen spurlos. Die Ernährungs- und Circulations-Bedingungen, sowie auch die Druckverhältnisse von Seiten der benachbarten Theile und die Veränderungen aller dieser Bedingungen können bei sonst normalen Verhältnissen nicht die Ursache des Schwundes der Inseln sein; das zeigten uns schon die Thierversuche, und das lehren uns auch die Beobachtungen an Mensohen, die bei verschiedenartigen Erkrankungen der Bauchspeicheldrüse nicht an Diabetes gelitten hatten. Ausserdem zeigte es sich schon damals, dass bei Thieren die Widerstandsfähigkeit der Langerhans'schen Inseln individuellen Schwankungen unterworfen ist.

In der Literatur findet sich, soweit mir bekannt ist, nur ein Versuch, einerseits die Veränderungen einiger Organe (Leber) bei Fehlen stärkerer schädlicher Einflüsse, 'andererseits das Fehlen solcher Veränderungen bei starken schädlichen Einflüssen, wie z. B. bei starkem Alkohol-Missbrauch, durch angeborene schwache, bezw. starke Widerstandsfähigkeit der Zell-Elemente dieser Organe, zu erklären (Kabanow).

Die Literatur über die pathologische Anatomie des Diabetes ist sehr umfangreich, doch finden sich in ibr nur sehr spärliche 
Angaben über die uns hier interessirenden Fragen. Eine Menge der verschiedenartigsten Affectionen der Bauchspeicheldrüse ist beschrieben worden, aber auch viele Mal (86) wurde bei den schwersten Formen der Krankheit die Drüse normal gefunden, und dieser Widerspruch, der besonders bei einem Vergleich der Sections-Befunde mit den experimentell erhaltenen Resultaten zu Tage trat, brachte die Autoren in grosse Verwirrung und liess sie wohl oft nach Durchsicht der Literatur an den Ausspruch von Goethes Faust denken: „Da steh' ich nun, ich armer Thor, und bin so klug, als wie zuvor." Auch in jüngster Zeit sagt z. B. Kahae: „Es ist daher eine vergebliche Mühe, die Pathologie des Diabetes auf den anatomischen Veränderungen aufbauenzu wollen." Lépine hat sich in letzter Zeit von seiner Theorie einer inneren Secretion von glykolytischem Ferment losgesagt und nimmt mit Töpfer und Dominicis als Ursache des Diabetes eine Störung der Verdauungsthätigkeit der Drüse an, was in krassem Widerspruch zu den bei den Transplantations-Versuchen eines Theils der Drüse unter die Haut erhaltenen Resultaten steht.

405 Fälle von Diabetes habe ich in der Literatur beschrieben gefunden mit anatomischen Untersuchungsdaten; in 4 Fällen davon werden die Inseln erwähnt, in weiteren 4 Fällen finden sich unklare Hinweise auf sie und in den übrigen Fällen fehlen jegliche Hinweise auf die Inseln.

Lemoine und Lannois fanden in ihren 4 Fällen eine Sklerose der Drüse, die von dem durch Bindegewebe zusammengedrückten und atrophischen Lymphapparat der Drüse ausging. Allem Anschein nach haben diese Autoren die Inseln, wie Renaut, für Lymphfollikel gehalten.

Kasahara fand in einem Falle von Diabetes eine geringe Anzahl Inseln.

Nauny n beschreibt einen Fall von Diabetes, wo die Inseln erhalten geblieben waren, doch handelte es sich nach Schultze um einen Diabetes nervösen Ursprungs.

Die ausführlichsten Angaben über die Inseln finden wir bei Dieckhoff. In mittelschweren Fällen von Diabetes fand dieser Autor eine Verringerung der Zahl der Inseln und im Fall XVIII, Diabetes von 11jähriger Dauer mit einem Zuckergehalt des Harns bis 6,4 pCt. bei einem Alkoholiker, konnte Dieckhoff 
in den Schnitten der schlaffen, zum Theil durch Fettgewebe ersetzten Drüse keine einzige Insel finden. Einerseits das Fehlen oder die Verringerung der Zahl der Inseln bei Diabetikern, andererseits das Erhaltenbleiben derselben bei anderen starken Affectionen der Drüse bei Nicht-Diabetikern in Betracht ziehend, kam Dieckhoff zu der Annahme, dass es gerade die Inseln sind, die eine specifische Function für Zucker-Ausscheidang besitzen. "In anderen Fällen ohne Diabetes (III und IV) war aber von den intertubulären Zellenhaufen ebenfalls keine Spur zu finden, und in einigen Fällen von Pankreas-Veränderungen mit Diabetes (z. B. Fall V, chron. Pankreatitis) war irgend eine Veränderung an diesen Zellhaufen nicht festzustellen. Wir sind also auf Grund unserer Untersuehungen in der That nicht in der Lage, der Anschauung beizupflichten, dass das Fehlen oder Zustandekommen des Pankreas-Diabetes wesentlich von den Verhältnissen der intertubulären Zellenhaufen abhängig sei.“"

Wenn wir aber diesen V. Fall näher analysiren, so erweist es sich, dass hier eine nicht ganz frische eiterige Pankreatitis vorlag. In den kurzen Daten über den Krankheits-Verlauf ist kein Wort über Zuckergehalt im Harne gesagt, und bei der Beschreibung des mikroskopischen Bildes sind die Inseln nicht erwähnt. Dagegen wurde in den Fällen III und IV von Dieckh off eine acute eiterige Pankreatitis beobachtet, und das Fehlen der Inseln und des Zuckers im Harn in diesen Fällen spricht nicht gegen die erwähnte Function der Inseln. Die Untersuchungen von v. Mering und Minkowsky haben ja gezeigt, dass bei diabetischen Thieren während der Dauer von Infections-Krankheiten und vor dem Tode die Zucker-Ausscheidung aufhört. Und Dieckhoff selbst sagt auf S. 132, dass trotz der schweren Veränderungen der Bauchspeicheldrüse intercurrente Infectionen, besonders Eiterungen, die Ursache des Fehlens der Zucker-Ausscheidung sein können. Die Fälle also, die nach Dieckhoff's Ansicht mit seiner Annahme von der Function der Inseln in Widerspruch stehen, thun das thatsächlich gar nicht, und seine Untersuchungen decken sich vollkommen mit den meinigen, was mir um so wichtiger ist, als Dieckhoff dabei zu einer anderen Anschaung gelangt ist.

Dieses vergebliche Suchen nach constanten und für die 
Diabetes charakteristischen Veränderungen wurde erstens bedingt durch den Umstand, dass die Forscher die Inseln ausser Acht liessen und die scheinbar gleichen Veränderungen der Dräse bei Diabetikern wie bei Nicht-Diabetikern nicht näher untereinander verglichen. Wenn das geschehen wäre, so wäre der Unterschied vermerkt worden, wie das bei Dieckhoff der Fall ist. Die ausschliessliche Untersuchung der Drüsen von Diabetikern, wo die Inseln manchmal ganz fehlen können, konnte auch nicht dazu beitragen, Licht in diese Frage zu bringen. Ein weiteres wichtiges Hinderniss war ferner das vollkommene Fehlen von Hinweisen auf die Langerhans'schen Inseln in den Lehrbüchern der normalen und pathologischen Histologie. Nur in dem Buch von Kölliker in dem v. Ebner bearbeiteten Abschnitte findet sich eine genaue und recht vollständige Beschreibung der Inseln, vollkommen befriedigende Abbildungen derselben und sogar die richtige Annahme, dass sie functionell als Blutdrüsen aufzufassen seien. Das Lesen der umfangreichen Literatur über den Bau des Pankreas war aber für Autoren, die z. B. nur 1 Fall zu beschreiben hatten, zu mühsam und auch unnütz. So lange eine genaue Beschreibung der Langerhans'schen Inseln mit Trennung derselben vom Verdauungs-Apparat nicht ihren Platz in den allgemein gebräuchlichen Lehrbüchern finden wird, so lange werden wahrscheinlich bei einer Beschreibung des mikroskopischen Bildes der Bauchspeicheldrüse die Inseln vergessen werden.

Ich werde nicht näher eingehen auf die Hypothesen von Cl. Bernard und seiner Anhänger, die nervöse Hyperproduction von Zucker, den herabgesetzten Zuckerverbrauch, bedingt durch das Fehlen von die Oxydation und die Ablagerung von Zucker begünstigenden Stoffen von Minkowsky, das glykolytische Ferment von Lépi ne und seinen Anhängern, von dem sich Lépine selbst später lossagte. Ich will nur darauf hinweisen, dass sich gegenwärtig die Facta zum Aufbau einer neuen Hypothese anzuhäufen beginnen. Es ist ja schon lange genug bekannt, dass der im Blute des Diabetikers circulirende Zucker von den Geweben nicht ausgenutzt werden kann und daher als giftige Substanz von den Nieren in intensivster Weise ausgeschieden wird. 
Das bringt mich auf den Gedanken, dass im Blut der Diabetiker der Zucker sich in anderer Form, als normal, befindet, dass er normal schwach an andere Stoffe gebunden ist, ich sage schwach gebunden, weil bis jetzt alle unsere Methoden diese Verbindung zerstörten. In letzter Zeit haben die Untersuchungen von Drechsel, Baldi, Henriques gezeigt, dass normal im Blut der Zucker nicht als solcher existirt, sondern in Form einer Verbindung mit Lecithin-ähnlichen Stoffen, einer Verbindung, die von Drechsel Jecorin genannt wird. Kolisch und Ritter von Stejskal aber gehen noch weiter. Sie nehmen an, dass das Jecorin noch schwach gebunden ist an Eiweissstoffe. Diese Autoren fanden, dass bei Diabetikern, wie auch bei normalen Leuten, sich mehr Jecorin als Zucker findet, stellten aber nicht fest, in wie weit die Verbindung des Jecorin mit Eiweissstoffen bei Diabetischen und unter normalen Verhältnissen sich gleichen. Der bekannte englischeChemiker Pav y aber, der sich viel mit Diabetes beschäftigt hat, $\mathrm{kam}$, in Betracht ziehend, dass bei gewissen Formen von Diabetes nur der vom Organismus selbst durch Spaltung der Eiweiss-Molecule hergestellte Zucker, ohne mit dem Harn ausgeschieden zu werden, verbrennen kann, zu der Annahme, dass Diabetes auf einer Störung der Assimilation beruhe, und dass bei dieser Krankheit der Organismus die Fähigkeit verliere, Zucker in Fett zu verwandeln und ebenso Zucker zu einem Eiweissglycosid za synthesiren.

Die Zukunft wird lehren, in wie weit Forschungen in dieser Richtung hin von Erfolg gekrönt sein könnten. Gegenwärtig jedoch kann man der Lösung dieser Frage näher treten, in dem man die Wirkung der Langerhans'schen Inseln in vitro et in vivo einem genauem Studium unterwirft. Früher wurde zu diesem $Z$ weck die Bauchspeicheldrüse in toto benutzt, jetzt aber haben wir in der Unterbindung des Ausführungsganges ein Mittel, die Inseln auf anatomischem Wege zu isoliren und den Chemismus dieser Elemente gesondert, mit Ausschaltung der Verdauungs-Fermente, zu studiren.

Diese anatomische Isolirung der Inseln gestattet aber auch in rationeller Weise eine Organo-Theraphie des Diabetes zu erproben. Bis jetzt haben alle Versuche, Diabetes durch Ein- 
führung der Bauchspeicheldrüse in toto auf verschiedenem Wege in den Organismus zu heilen, keinerlei einigermaassen sichere Resultate gegeben (Battistini, Blumenthal, Brown-Sequard, Capparelli, W. Hale White, W. A. Wills). Da es sehr schwierig ist, in grösserer Quantität Drüsen zu beschaffen, in denen nur die Inseln erhalten sind, so könnte man sie durch Drüsen von neugeborenen Thieren, z. B. von Kälbern, ersetzen, bei denen die Inseln im Verhältniss zum Verdauungs-Apparat sehr gut entwickelt sind; dazu käme noch, dass die BauchSpeicheldrüse der Neugeborenen wenig befähigt ist zur Verdauungsarbeit und man also hoffen darf, dass ihre Verdaungssäfte die Wirkung der von den Inseln ausgearbeiteten Stoffe nicht beeinträchtigen wird. Auf alle Fälle darf man hoffen, dass es der nächsten Zukunft vorbehalten ist, die Frage zu lösen, ob es auf diesem Wege gelingt, die Leiden der Diabetiker zu lindern.

Zum Schlusse ist es mir eine angenehme Pflicht, meinen verehrten Lehrern, den Herren Professoren K. N. Winogradow and J. P. Pawlow und dem Herrn Privat-Docenten A. J. Moissejew, meinen herzlichsten Dank auszusprechen für das Interesse, das sie meiner Arbeit entgegengebracht haben und für ihre Bereitwilligkeit, mir jederzeit mit Rath und That beizustehen, die ich nicht selten Gelegenheit hatte, zu benutzen.

\section{Nachtrag.}

In No. 41 der Wiener klinischen Wochenschrift vom Jahre 1901 findet sich die Arbeit von Professor Weichselbaum und Dr. Tangl.

Diese Autoren untersuchten die Bauchspeicheldrüse des Menschen bei Diabetes und verschiedenen anderen Erkrankungen dieser Drüse und bestätigten vollkommen die von mir erhaltenen Resultate. Die Zeichnungen, die der Arbeit beiliegen, gleichen ungemein meinen Zeichnungen No. 14 und 15.

\section{Verzeichniss der wichtigsten Literatur. ${ }^{1}$ )}

Arnozan et Vaillard: Gazette med. de Paris, 1881, S. 360; Journal de médécine de Bordeaux, 1880-1881, S. 584; Archives de physiologie, 1884, S. 287.

1) Die von mir benutzte Literatur beträgt etwa die Grösse von ungefähr 200 Arbeiten. Hier führe ich nur die wichtigsten an. Andere kann man in Oser's Monographie (Nothnagel's Handbuch) finden. 
Baldi: Areh. f. Anat. u. Phys., phys. Abth., Suppl.-Band, 1887, S. 100.

Battistini: Therapeut. Monatshefte, VII, 1833, S. 494.

Böhm und Dawydow: Lehrbuch der Histologie des Menschen. Russ. Uebers., Moskau, 1897 u. 1899.

Bernard Claude: a) Memoire sur le pancréas et sur le rôle du suc pancréatique, Paris, 1856; b) Leçons de pathologie expérimentelle, Paris, 1872 ; c) Leçons sur le diabète et la glycogenèse animale, Paris, 1877.

Bizzozero, G. und Vassale, G.: Dieses Archiv, Bd. 110, 1887, S. 155. B lumenthal: Zeitschr. f. physikal. u. diät. Ther., 1898.

Brown Séquard et d'Arsonwal: Arch. de phys., 1891, S. 491.

Capparelli: Archives ital. de Biol., Bd. 18 u. 21.

Diamare: Internat. Monatsschr. f. Anatomie u. Phys, 1899.

Dieckboff, Christ.: Beiträge zur wissensch. Medicin. Festschr, gewidm. Theodor Thierfelder. Leipzig, 1895.

Dogiel: Archiv f. Anat. u. Phys., anat. Abth., 1893, S. 117.

Drechsel: Journal für prakt. Chemie, Bd. 33, 1886, S. 425.

v. Ebner: a) Arch. f. mikrosk. Anat., Bd. 8; b) Kölliker's Handbuch dẹr Gewebelebre d. Mensehen, Leipzig, 6. Aufl., 1899.

Galeotti: Internat. Monatsschr. f. Anat. u. Phys., 1895.

Gartier: Die Bauchspeicheldrüse der Früchte und der Neugeborenen des Menschen. Inaug.-Dissert., St. Petersburg, 1900.

Gibbes: The Quarterly Journal of mikrosk: Science, V. 24, 1884, S. 183. Gley: Comptes rendus de la Soc. de Biol. de Paris, 1891 u. 1892.

Hansemann: Zeitschr. f. klin. Med., Bd. 26.

Hedon: a) Arẹhiv. de méd. expér., 1891, S. 33, 311, 527; b) Archives de phys., $1891,1892,1894$; c) C. R. de la Soc. de Biol, 1891, 1892 ; d) C. R. de l'Acad. des Sciences de Paris, 1893, S. 238 u. 649 .

Heidenhain, R.: Pflüger's Archiv f. ges. Phys., Bd. 9:

Heidenhain, M.: Zeitschr. f. wissensch. Mikroskopie, Bd. 13, 1896.

Henriques: Zeitschr. f. physiol. Chemie, Bd. 23.

Iwanowsky, N. P.: Zur Lehre von der visceralen Syphilis. Inaug.-Diss. St. Petersburg, 1871.

Jarotzky: Ueber die Veränderungen der Grösse und der Structur der Pankreaszellen bei einigen Arten von Hunger.

Kabanow: Ueber die Rolle der Erblichkeit in der Aetiologie der Krankheiten der inneren Organe. Moskan, 1888.

Kasabara: Dieses Archiv, Bd. 143, S. 111.

Katz und Winkler: Archiv f. Verdauungs-Krankheiten, 1898.

Kolisch, R. und Ritter v. Stejskal: Wien. klin. Wochenschr., 1897.

Kudrewetzky: Zeitschr. f. Heilkunde, Bd. 13.

Kühne, W. und Lea, A.: Untersuchungen aus dem physiol. Institute der Universität Heidelberg, Bd. 2, 1882. 
Laguesse: a) Journal de l'anat. et de physiologie, 1894, S. 591 и. 79 , 18.96; b) C. R. de la Soc. de Biol., 1899.

Langerhans, Paul: Beiträge zur mikroskop. Anatomie der Bauchspeicheldrüse. Inaug.-Dissert., Berlin, 1869.

Lawdowsky und 0 wjannikow: Lehrbuch der mikroskop. Anatomie der Menschen und Thiere. St. Petersburg, 1892.

Lefas: Archives génèrales do med., 1900.

Lemoine, G. et Lannois, M.: Arehives de med. exper., 1891.

Lépine, R.: a) Berliner klin. Wochenschr., 1891; b) Lyon med., 1899; Bd. 90.

Lewatschew: Arehiv f. mikr. Anatomie, Bd. 26.

Litten: Charité-Annalen, 1878, S. 181.

Maximow, A.: Russiseh. Archiv der Pathol., Bd. 1.

Mandelstamm: Zur Pathologie der Bauchspeicheldrüse bei den acuten Infections-Krankbeiten. Inaug.-Dissert. Kasan, 1873.

Mankowsky: Zur. Mikrophysiologie der Bauchspeicheldrüse. Inaug.-Diss. Kiew, 1900, und kurz Arch. f. mikr. Anatomie, Bd. 59, S. 286.

Martynow, A.: a) Ueber die Cirrhosen der Bauchspeicheldrüsen. Verhandl. der Moskatuer therapeut. Gesellsch, 1898; b) Die Chirurgie der Bauchspeicheldrüse.

v. Mering und Minkowski: Arehiv für experim. Pathol., Bd. 26, 1890 S. $371-387$.

Mink owski, O.: Ebendaselbst, Bd. 31, 1893, S. 85-189.

Mouret: C. R. de la Soc. de Biol., 1894 u. 1895.

Munk: Schmidt's Jabrbücher, Bd, 144.

Naunyn: Ref. nach Schultze.

Oser: Die Erkrankungen des Pankreas. Nothnagel's Handbuch der spec. Path. u. Ther., Wien, 1898, Bd. 18, Th. II.

Pawlow, J. P.: Pflüger's Archiv, Bd. 16.

Podwyssotzky, W.: Kiewsche Universitäts-Nachrichten, 1881.

Popielsky, L.: a) Ueber die Secretion-unterdrückenden Nerven der Bauchspeicheldrüse, Inaug.-Dissert., Petersburg, 1896; b) Ueber das reflectorische Centrum der Bauchspeicheldrüse. Arch. f. Physiol., 1901.

Pugnat: Ärehives de l'anat. et de physiologie, 1897.

Remy et Showe: C. R. de la Soc. de Biol., 1882.

Renaut: a) O. R. Ac. d. Sciences de Paris, 1879 ; b) Arch. de phys, 1881. Ribbert: Centralbl. f. klin. Med., Bd. 1, S. 385 .

Rodionow: Zur pathol. Auat. der Bauchspeicheldrüse bei den allgem. Infections-Krankheiten. Inaug.-Dissert., St. Petersburg, 1883.

Saviotti: Arch. f. mikr. Anat., Bd. 5, S. 104.

Schlesinger: Dieses Archiv, Bd. 154.

Schultze, W.: Archiv f. mikr. Anat., Bd. 56.

Seim: Volkmann's Sammlung klinischer Vorträge, 1888, No. 313-314. 


\section{6}

St atkewitsch: Arch. f. exper. Path., Bd, 33.

Schabad: Zur Frage vom pankreatischen Diabetes. Inaug.-Diss., Moskau, 1895.

Stschastny: Russisch. Archiv der Pathologie, 1898, Bd. 5, S. 697.

Ssobolew: Centralbl. f. allg. Path. U. path. Anat, 1900, No. 6 u. 7, vorl. Mittheilung.

Thiroloix: a) Le diabète pancréatique, Thèse de Paris, 1892; b) Gazette des bôpitaux, 1894.

Treiberg: Zur Diagnostik u. Therapie d. Pankreas-Cysten. Inaug.-Diss., St. Petersburg, 1888.

Tschassownikow: Die Nachrichten der. Universität Warschau, 1900, No. 3, 4, 5 .

Wassiljew: Pathologisch-anatomisches Bild der Magenkatarrhe bei kleinen

Kindern. Inaug.-Dissert., St. Petersburg, 1900.

\section{Erklärung der Abbildungen auf Tafel IV u. V.}

Die Zeichnungen 8 und 10 wurden bei einer Vergrösserung von 70 (Zeiss, Ob. AA. oc. 3), die Zeichnung 4 bei einer Vergrösserung von 120 (Ob. AA. oc. 5), 9 und 13 bei einer Vergrösserung von 535 (DD. oc. 5), 6 und 7 bei einer Vergrösserung von 1180 ( $\tau_{1}^{\frac{1}{2}}$ Oel-Imm. oc. 5), alle übrigen bei einer Vergrösserung von 325 (DD. oc. 3) angefertigt.

Die für alle Zeichnungen geltenden Abkürzungen: il = Langerhans'sche Inseln, $p \mathrm{t}=$ Drüsenröhrchen des Pankreas, $c=$ Capillargefäss, $d=$ Ausführungsgang, $\mathrm{m}=$ Mitose, $\mathrm{g} Z=$ grosse körnige Zelle, $\mathrm{kZ}=$ kleine Zelle in den Inseln des Kaninchens.

Fig. 1. Normales Kaninchen. In der Insel sieht man die Anorduung der Zellen zu Colonnen, die von einander durch Capillargefässe getrennt sind, und die Eintheilung der Zellen in 2 Kategorien.

Fig. 2. Bauchspeicheldrüse eines Kaninchens, $7 \frac{1}{2}$ Tage nach der Unterbindung des Ausführungsganges. Im Centram eine von atrophirenden Drïsenröbrchen umgebene Insel.

Fig. 3. Drüse eines Kaninchens 20 Tage nach der Unterbindung des Ausführungsganges; im Centrum eine von 3 Seiten von fibrillärem Bindegewebe umgebene Insel, nur rechts sind feine Ausfübrungs. gänge mit hyperplastischem Epithel sichtbar.

Fig. 4. Pankreas eines Kaninchens 200 Tage nach der Unterbindung des Ausführungsganges; inmitten des theils fibrösen, theils Fettgewebes liegen die gut erhalten gebliebenen Inseln recht nahe bei einander.

Fig. 5. Drüse eines Hundes 20 Tage nach der Unterbindung des Ausführangsganges, im Centrum eine gut erhalten gebliebene Insel, die von Drüsenröhrchen mit fettig degenerirten Zellen umgeben ist; diese Zellen liegen unregelmässig, uad es ist schwer, die Drüsenröhrchen als solche zu erkennen. 
Fig. 6. Eine Inselzelle eines Hundes nach 2 tägigem Hungern. Im Protoplasma viele Körnchen, die sich durch Fuchsinsäure färben liessen; die gröberen Körnchen liegen an der Peripherie der Zelle. Härtung und Färbung nach Altmann.

Fig. 7. Inselzelle eines Hundes nach Exstirpation von mehr als $\frac{2}{3}$ der Drüse und verstärkter Fütterung mit Kohlehydraten plus ZuckerInjection in das Blut. Die Zelle ist im Volumen verkleinert, ihr Protoplasma enthält wenig Körnchen, von denen einige Fetttröpfehen darstellen.

Fig. 8. Pankreas einer menschlichen Frucht von $31 \mathrm{~cm}$ Lãnge. In der Drüse fällt die grosse Zahl der Inseln stark auf, die in Folge einer schwachen Entwickelung des Lymphapparates sehr dicht bei einander liegen; a eine feincalibrige Arterie.

Fig. 9. Ein Theil der Insel eines 5 Tage alten Meerschweinchens. Hier sieht man 2 Zellen in indirecter Theilung begriffen. Die Insel enthält verbältnissmässig viel grosse körnige Zellen, deren Kerne sich nur wenig von den Kernen der kleineren Zellen unterscheiden.

Fig. 10. Pankreas eines 3 Wochen alten Kaninchens, Gefāsse mit Gelatinemasse injicirt. Im Centrum eine aus einem Geflecht von Zellcolonnen bestehende Insel; zwischen den Colonnen breite Capillargefässe, die von ein wenig geschrumpfter Injectionsmasse angefüllt sind.

Fig. 11. Sklerose der Bauchspeicheldrüse. In der Mitte eine gut erhalten gebliebene Langerbans'sche Insel. Um sie herum Reste der Drüsenröhrchen (pt) und der Ausführungsgänge inmitten recht zellreichen Bindegewebes.

Fig. 12. Der untere Theil derselben Stelle wie in Fig. 11 bei stärkerer Vergrösserung; der Unterschied zwischen den atrophirenden Zellen der Drüsenröhrchen und den gut erhaltenen Inselzellen ist deutlich sichtbar (B. I. Abschnitt Fall 9).

Fig. 13. Sypbilis hereditaria (B. I. Abschnitt, Fall 18). In der Mitte eine gut erhaltene Langerhans'sche Insel, umgeben von fibrillärem Bindegewebe. Viele Fibrillen dieses Gewebes sind verdickt und hyalin degenerirt (hf). Die Drüsenröhrchen und die Ausführungsgänge sind geschwunden.

Fig. 14. Pankreas bei Diabetes (B. Fall 3). In der Mitte eine im Volumen bedeutend verkleinerte Insel. Die Kerne sind schwächer als normal gefärbt, stellenweise fehlt ihr Protoplasmasaum. Bei den Zellen, wo dieser Saum erbalten ist, sind in ibm feine dunkle Körnchen - Fett - zu sehen. $v-$ Vacuolen, durch Sehwund der Zellen entstanden. Das Capillarnetz ist gut erhalten.

Fig. 15. Pankreas bei Diabetes (B. II. Abschnitt Fall 9). In der Mitte eine im Volumen verkleinerte Insel, die aus kleinen Zellen mit 
kleinen, eckigen, intensiv gefärbten, pyknotischen Kernen bestebt ( $(\mathrm{pk}), \mathrm{k}, \mathrm{k}$.wei recht gut erhaltene Kerne. Um die Insel herum wenig veränderte Drüsenröhrchen.

Fig. 16. Bauchspeicheldrüse einer Taube. In der Mitte eine Langerhanssche Inșel, c Capillargefäss.

\section{V. \\ Zur Anatomie und Pathogenese der Divertikel der vorderen Oesophagus-Wand.}

(Aus dem Pathologischen Institut der Universität Bern.)

Von

Max Hausmann, med. pract., ehemal. 2. Assistenten am Pathologischen Institut zu Bern.

(Hierzu Taf. VI.)

Die Divertikel des Oesophagus haben ihre erste ausführliche und auch abschliessende Schilderung durch Zenker und Ziemssen $^{33}$ erfahren. Die beiden Autoren begründeten damals die seither überall adoptirte Eintheilung in Pulsions- und TractionsDivertikel, d. h. Divertikel, die einem Drucke von innen oder einem Zuge von aussen ihre Entstehung verdanken. Ihre PulsionsDivertikel waren zugleich charakterisirt dureh den constanten Sitz an der Grenze von hinterer Pharynx- und Oesophagus-Wand und durch ihre klinische Wichtigkeit, die Tractions-Divertikel durch ihr regelmässiges Vorkommen an der Vorderwand (am häufigsten an deren mittlerem Drittel) und ihre relative klinische Harmlosigkeit. So war die Unterscheidung eine äusserst prägnante, sie verfehlte auch nicht, entgegen Zenker, schematisch zu werden. Da die Tractions-Divertikel an der Vorderwand sitzen, war man bald geneigt, umgekehrt jedes Divertikel der vorderen Oesophagus-Wand als Tractions-Divertikel anfzufassen. In der neueren Special-Literatur freilich tauchten da und dort Mittheilungen auf, die dem Schema nicht entsprachen, und die beiden Autoren Brosch und Starck ${ }^{78}$, die sich in neuester 